\title{
QUANTUM APPROACH TO THE GRAVITATIONAL WAVES
}

\author{
Sebastiano Tosto \\ Retired Physicist, ENEA Casaccia Via Anguillarese 00123, Italy
}

Received 2013-12-05; Revised 2014-01-05; Accepted 2014-01-06

\begin{abstract}
The study proposes a quantum approach to explain existence and main features of the gravitational waves radiated by an isolated two body orbiting system. The quantum eigenvalues of such a system are calculated with the help of the space-time quantum uncertainty, without additional hypotheses via an "ab initio" model. The approach is deliberately non-relativistic: The model does not implement any relativistic assumption and shows that the mere quantum approach is enough to get energy loss and orbit radius contraction coincident with that early inferred by Einstein. However the outcomes of the model also show features typical of the quantum systems, which are purposely discussed.
\end{abstract}

Keywords: General Relativity, Gravitational Waves, Quantum Uncertainty

\section{INTRODUCTION}

The general relativity is commonly regarded as the natural starting point and master tool to describe the structure and evolution of the universe; in effect the modern cosmology was born with the early models of inflationary universe of De Sitter implementing the cosmological constant. The theoretical basis to describe the evolution of the universe was successively provided by the Friedmann solution of the Einstein gravity field equations. The parallel progress of the quantum theory, historically born in the conceptual frame of the particle physics, allowed valuable understanding about the nuclear processes that generate the energy irradiated by the stars; also, explaining the abundance of chemical elements of the universe (Clayton, 2007; Seeger et al., 1965) was probably the most successful contribution of the quantum mechanics to the cosmology. While the relativity opened the pathway to a new class of unpredicted phenomena, e.g., the gravitational lensing (Cohen, 1988; Blandford and Narayan, 1992) and the generation of gravitational waves (Landau and Lifshitz, 1987; Thorne, 1973; Marck and Lasota, 1997), still today the quantum mechanics remains in the common sense the science of the microscopic world. On the one side the quantum physics is required to explain specific topics like the Hawking entropy (Hartle and Hawking, 1976), the vacuum polarization at the event horizon of black holes (Peskin and Schroeder, 1995), the origin of the cosmic microwave background radiation (Dinculescu, 2013) and the vacuum energy density (Milonni, 1994); also, the standard model has been implemented to attempt explaining the dark energy and dark matter (Jungmann et al., 1996). On the other side, however, the contribution of quantum mechanics seems mostly confined to topics anyway related to the physics of elementary particles. This limitation is partially due to the difficulty of transferring the information on nanoscale phenomena to the knowledge of the whole universe; yet it surely rests also on the conceptual difficulty of implementing the weird concepts of nonreality and nonlocality to understand the objects which the universe is made of. Some hint to overcome this information gap comes from the string cosmology (Lidsey et al., 2000), formulated in order to obtain an inflationary model of universe; assuming that the usual four-dimensional universe is actually a particular brane in the frame of a higher dimensional space, the string theory formulates the metric of the model at various energy scales. According to this theory the gravitons that carry the gravitational interaction should be vibrations of closed strings (Rothman and Boughn, 2006; Will, 1998). These new theories share the peculiarity of requiring a large number of extra-dimensions, e.g., 26 for bosonic string theories (Polchinski, 1998) or 10 for superstring theories (Polchinski, 2001) or 11 according to the socalled M-theory (Duff, 1996), whose existence is still today not definitively proven by the experimental

(C) 2013 Sebastiano Tosto. This open access article is distributed under a Creative Commons Attribution (CC-BY) 3.0 license which permits unrestricted use, distribution, and reproduction in any medium, provided the original work is properly credited. DOI: 10.3844/pisp.2013.135.151 
evidence; moreover the lack of an agreement on the number of extra-dimensions, still unfixed itself, makes questionable the physical meaning of its actual reality. Besides these attempts, therefore, the direct involvement of quantum physics and its concepts to relativistic problems like the generation of gravitational waves and the light beam bending would be useful. An example of possible problem is suggested in this respect by the quantum interaction of atoms with radiation; the electron energy levels absorb electromagnetic energy and get an excited state, from which they successively decay by emission of radiation. The general relativity describes the emission of gravitational waves calculating $\partial \varepsilon / \partial t<0$ and $\partial \mathrm{r} / \partial \mathrm{t}<0$ : Energy is lost by an orbiting system, whose orbital radius therefore shrinks as a function of time. A possible question is the following: Could an orbiting system and its energy release be regarded as a resonance driven reversible phenomenon? In other words: Could the gravitational waves emitted by a given system with appropriate frequency be absorbed by another orbiting system correctly tuned, which is thus raised to an excited state with higher energy and larger orbit radius? Further questions are of course possible, for instance: Let a two body orbiting system be such that its size and total mass fulfil the black hole condition; could in principle such a system emit gravitational waves? The quantum mechanics appears to be an appropriate tool to investigate such kind of theoretical problems, which remain however still today deeply rooted into the realm of the gravitational theories only. Some papers recently published have shown the chance of a more substantial incorporation of quantum concepts into the typical domain of the general relativity implementing the spacetime quantum uncertainty only. For instance it has been possible to replicate the solution of the Friedmann equation via the statistical formulation of the quantum uncertainty equations early introduced as attempt to describe quantum systems (Tosto, 2013a); in this way even the Lorentz transformations and the Hawking entropy have been inferred as a corollary.

The present paper proposes an attempt to implement directly quantum ideas into two large scale problems of cosmological valence, the gravitational waves and the light beam bending. Of course this does not mean merely replicating known results of the relativity; rather, it allows enriching our current understanding on these topics with the help of quantum ideas.

The following "ab initio" model introduces an approach deliberately non-relativistic: The aim is to show that relativistic results are nevertheless easily obtained plugging considerations of classical physics into the quantum world via the space-time uncertainty only. In particular the approach outlined below aims to show that even the naïve quantum model of a gravitational system with circular orbits is enough to infer existence and main properties of gravitational waves; despite this topic is typically regarded as a relevant outcome of the general relativity only, the following considerations show that instead the gravitational waves can be in fact regarded as a quantum phenomenon.

\section{QUANTUM BACKGROUND}

Physicists believe unsatisfactory a quantum theory based on the wave function $\psi$ without direct physical meaning, e.g., (Leonhardt and Paul, 1995); indeed $\psi^{*} \psi$ only has the statistical meaning of probability density and contains the maximum information obtainable about a physical system. Moreover also the Wigner distribution function (Levanda and Fleurov, 2001), although providing significant information about the quantum states, presents conceptual difficulties: It cannot be really regarded like a probability distribution in the classical sense, rather it is a quasi-probability that can take even negative values; moreover it can represent the average value of an observable but not, in general, also its higher power moments. Both these difficulties are bypassed exploiting the statistical formulation of the quantum uncertainty, regarded as a fundamental assumption itself; it reads in one space dimension:

$\Delta \mathrm{x} \Delta \mathrm{P}_{\mathrm{x}}=\mathrm{n} \hbar=\Delta \varepsilon \Delta \mathrm{t}$

The second equality is formally obtained from the former rewriting $\left(\Delta \mathrm{x} / \mathrm{v}_{\mathrm{x}}\right)\left(\mathrm{v}_{\mathrm{x}} \Delta \mathrm{P}_{\mathrm{x}}\right)=\mathrm{n} \hbar$ with $\mathrm{v}_{\mathrm{x}}=\Delta \mathrm{x} / \Delta \mathrm{t}$ and $\Delta \varepsilon=\mathrm{v}_{\mathrm{x}} \Delta \mathrm{P}_{\mathrm{x}}$; the number $\mathrm{n}$ of allowed states is uniquely defined and arbitrary like the sizes of the uncertainty ranges. Equation (1 and 2) compel the positions:

$$
\mathrm{x} \rightarrow \Delta \mathrm{x} \quad \mathrm{P}_{\mathrm{x}} \rightarrow \Delta \mathrm{P}_{\mathrm{x}} \quad \mathrm{t} \rightarrow \Delta \mathrm{t} \quad \varepsilon \rightarrow \Delta \varepsilon
$$

The Equation (1) are the unique assumption of the model, no further hypothesis is necessary besides that of waiving the random local values of the dynamical variables, considered random, unknown and unpredictable; in other words, the positions (2) are compliant with the idea that coordinates do not exist a priori in nature, being only mathematical artifices used in describing the reality. The quantum eigenvalues show indeed that they play no role in the formulation of the observables. The Equation (1) enable the quantum properties of particles to be inferred considering the definitions of the physical observables of interest only, 
without solving the pertinent wave equations. The conceptual features of the present quantum model and its extension to the relativity have been described elsewhere (Tosto, 2012; 2013a); here two remarks only are shortly sketched to highlight why the positions (2) have prospective interest also in cosmology. First of all, these positions waive introducing a particular reference system. Write for instance $\Delta \mathrm{x}=\mathrm{x}_{1}-\mathrm{x}_{0}$ in any $\mathrm{R}$, with $\mathrm{x}_{\mathrm{O}}$ and $\mathrm{x}_{1}$ boundary coordinates arbitrary and unknown. Either of them, say $x_{0}$, defines the distance of the range from the origin of $\mathrm{R}$, whereas $\mathrm{x}_{1}$ defines its size; if however $\mathrm{x}_{\mathrm{o}}$ is arbitrary and indefinable, then $\mathrm{R}$ is indefinable as well. Hence, once accepting the positions (2), all reference systems are equivalent as concerns the consequences of the Equation (1) on the basis of uncertainty ranges only; of course an analogous reasoning holds for all ranges. This conclusion is clearly compliant with one basic assumption of the relativity. Moreover, the fact that $\mathrm{x}_{1}$ is arbitrary as well, means that the timespace scale of validity of Equation (1) is not fixed; since this holds for all ranges, this means that the present model holds regardless of the nano-or micro-or macro-scale of space, time, energy and momentum of the concerned quantum system.

\section{QUANTUM MODEL OF A TWO-BODY GRAVITATIONAL SYSTEM}

Consider in an arbitrary reference frame $\mathrm{R}$ an isolated orbiting system formed by two classical particles having total mass $\mathrm{M}=\mathrm{m}_{1}+\mathrm{m}_{2}$ and interacting via the potential field $\varphi$. Introducing the reduced mass $\mu=\mathrm{m}_{1} \mathrm{~m}_{2} / \mathrm{M}$ and the angular momentum $\mathrm{J}$ of the system, both expected for interacting particles, the classical energy equation $\varepsilon=\mathrm{p}^{2} / 2 \mu+\varphi$ is $\varepsilon=\mathrm{p}_{\mathrm{r}}^{2} / 2 \mu+\mathrm{J}^{2} / 2 \mu \mathrm{r}^{2}+\varphi$, where $\mathrm{r}$ is the distance of $\mu$ from the fixed origin of R. Let us expand $\varphi$ in series of powers of $r$ to determine the effect of the perturbation on the system induced by the interaction; as here we are interested in particular to a field tending to a constant at infinity, write without loss of generality $\varphi=\sum_{\mathrm{k}=0}^{\infty} \alpha_{\mathrm{k}} \mathrm{r}^{-\mathrm{k}}$ with appropriate coefficients $\alpha_{\mathrm{k}}$ that specify the pertinent kind of potential. Put $\Delta \varepsilon=\varepsilon-\alpha_{0}$; the classical energy equation reads at the second order of the potential series development:

$$
\Delta \varepsilon=\frac{\mathrm{P}_{\mathrm{r}}^{2}}{2 \mu}+\frac{\mathrm{b}}{\mathrm{r}^{2}}+\frac{\mathrm{J}^{2}+2\left(\alpha_{2}-\mathrm{b}\right) \mu}{2 \mu \mathrm{r}^{2}}+\frac{\alpha_{1}}{\mathrm{r}}+\cdots
$$

The classical energy range $\Delta \varepsilon$ defined by $\varepsilon$ and the constant potential term $\alpha_{0}$ is in principle exactly definable; for instance nothing prevents putting $\alpha_{\mathrm{o}}=0$ if in particular the potential energy is set equal to zero at infinity. Moreover the local dynamical variables require specifying a reference system, e.g., whose origin coincides with the orbit centre or orbit focus.

At this point let us take into account the condition compelled by Equation (1) on the conjugate dynamical variables; this requires implementing the positions (2), according which $\mathrm{r} \rightarrow \Delta \mathrm{r}$ and $\mathrm{p}_{\mathrm{r}} \rightarrow \Delta \mathrm{p}_{\mathrm{r}}$. Being now $\Delta \mathrm{p}_{\mathrm{r}}=$ $\mathrm{n} \hbar / \Delta \mathrm{r}$ the Equation (3) turns at the second order into:

$$
\Delta \varepsilon=\frac{(\mathrm{n} \hbar)+2 \mathrm{~b} \mu}{2 \mu \Delta \mathrm{r}^{2}}+\frac{\mathrm{J}^{2}+2\left(\alpha_{2}-\mathrm{b}\right) \mu}{2 \mu \Delta \mathrm{r}^{2}}+\frac{\alpha_{1}}{\Delta \mathrm{r}}
$$

Note that the positions (2) do not introduce a mere change of notation. The range $\Delta \varepsilon$ encloses the possible $\varepsilon$ related to all of the allowed values of local dynamical variables $r$ and $p_{r}$ within the respective $\Delta r$ and $\Delta p_{r}$, i.e., the size of $\Delta \varepsilon$ corresponds to and is consequence of the sizes of these latter: The classical equation linking dynamical variables exactly known turns into the connection between uncertainty ranges of these dynamical variables, whose local values included within them are now completely arbitrary and unknown by definition. This holds also for the angular momentum $\mathrm{J}$, which is expected to change within its consequent range $\Delta \mathrm{J}$. The uncertainty ranges introduced without any hypothesis about their boundary values waive the existence of a specific reference system, which becomes from now on arbitrary and indeterminable itself; in effect this is demonstrated below. Rewrite identically as follows the equation resulting from the positions (2):

$$
\begin{aligned}
& \Delta \varepsilon=\frac{1}{2 \mu}\left(\frac{\mu \alpha_{1}}{\sqrt{(\mathrm{n} \hbar)^{2}+2 \mathrm{~b} \mu}}+\frac{\sqrt{(\mathrm{n} \hbar)^{2}+2 \mathrm{~b} \mu}}{\Delta \mathrm{r}}\right)^{2} \\
& -\frac{1}{2} \frac{\mu \alpha_{1}^{2}}{(\mathrm{n} \hbar)^{2}+2 \mathrm{~b} \mu}+\frac{\mathrm{J}^{2}+2\left(\alpha_{2}-\mathrm{b}\right) \mu}{2 \mu \Delta \mathrm{r}^{2}}
\end{aligned}
$$

Owing to the different physical meaning of $\Delta \varepsilon$ in the classical Equation (3) and in the quantum Equation (4) by effect of the positions (2), the symbol $\Delta$ implements the consequent idea that $\mathrm{n}$ is arbitrary and not uniquely definable; $\Delta \varepsilon$ symbolizes an arbitrary range of energies corresponding to the respective values of $n$, in agreement with the concept of quantum uncertainty previously introduced. Otherwise stated, $\Delta \varepsilon$ defines a set of quantized values $\varepsilon(n)$. At this point minimize $\Delta \varepsilon$ putting equal to zero the positive squared term, which means searching the set of minimum energy values in it included; one finds: 


$$
\begin{aligned}
& \Delta \mathrm{r}=-\frac{(\mathrm{n} \hbar)^{2}+2 \mathrm{~b} \mu}{\mu \alpha_{1}} \quad \Delta \varepsilon=\frac{\mu \alpha_{1}^{2}}{2} \frac{\mathrm{J}^{2}+2\left(\alpha_{2}-\mathrm{b}\right) \mu}{\left((\mathrm{n} \hbar)^{2}+2 \mathrm{~b} \mu\right)^{2}}-\varepsilon_{0} \\
& \varepsilon_{0}=\frac{1}{2} \frac{\mu \alpha_{1}^{2}}{(\mathrm{n} \hbar)^{2}+2 \mathrm{~b} \mu}
\end{aligned}
$$

Note that in principle both $(n \hbar)^{2}+2 b \mu>0$ and $(n \hbar)^{2}+$ $2 \mathrm{~b} \mu<0$ are allowed, being anyway $\Delta \mathrm{r}$ real; these chances depend on $n \hbar$ and on the sign of $b$, on which no hypothesis has been made. Taking for instance $\Delta \mathrm{r}$ positive by definition, the inequalities require respectively $\alpha_{1}<0$ and $\alpha_{1}>0$. Accordingly the sign of the second addend of the Equation (4) depends upon $\mathrm{b}$, that of the third addend upon $\alpha_{2}$-b with respect to $\mathrm{J}^{2} / 2 \mu$ and so on; by consequence $\Delta \varepsilon$ can consist of both negative binding energies or positive nonbinding energies. This shows that both attractive and repulsive gravitational potentials are in principle allowed in the present model, as already found in (Tosto, 2013b). In this study $\alpha_{1}<0$ with $(\mathrm{n \hbar})^{2}+2 \mathrm{~b} \mu>0$ only is of interest and will be next considered.

To assess the second Equation (5), it is necessary to find the quantum expression of $\mathrm{J}^{2}$ and then determine the series coefficients $a_{1}$ and $b$. Calculate first the component $J_{w}=r \times p \cdot w$ of $J=r \times p$ along the arbitrary unit vector $w$. Once again the positions (2) compel $\mathrm{r} \rightarrow \Delta \mathrm{r}$ and $\mathrm{p} \rightarrow \Delta \mathrm{p}$ to calculate the number 1 of states consistent with the ranges $\Delta \mathrm{r}$ and $\Delta \mathrm{p}$ physically allowed to the particle. So $\mathrm{J}_{\mathrm{w}}=(\Delta \mathrm{r} \times \Delta \mathrm{p}) \cdot \mathrm{w}$ yields $\mathrm{J}_{\mathrm{w}}=$ $\Delta \chi \times \Delta \mathrm{p}$, where $\Delta \chi=\mathrm{w} \times \Delta \mathrm{r}$. Therefore $\mathrm{J}_{\mathrm{w}}=0$ if $\Delta \mathrm{p}$ and $\Delta \chi$ are orthogonal; else $\Delta \chi \cdot \Delta \mathrm{p}=(\Delta \mathrm{p} \cdot \Delta \chi / \chi) \chi$ with $\chi=$ $|\Delta \chi|$ yields $\pm \Delta \mathrm{p}_{\chi}=\Delta \mathrm{p} \cdot \Delta \chi /|\Delta \chi|$ and thus $\mathrm{J}_{\mathrm{w}}= \pm \Delta \chi \Delta \mathrm{p}_{\chi}$, i.e., $J_{\mathrm{w}}= \pm$ lh according to Equation (1). In principle one component of $\mathrm{J}$ only is knowable: Repeating the same approach for another component would trivially mean changing w. So the average values $\left\langle\mathrm{J}_{\mathrm{x}}^{2}\right\rangle,\left\langle\mathrm{J}_{\mathrm{y}}^{2}\right\rangle$ and $\left\langle\mathrm{J}_{\mathrm{z}}^{2}\right\rangle$ calculated in the same way should be equal; hence the $\mathrm{x}$ - and $\mathrm{y}$ - and $\mathrm{z}$-components averaged over the possible states summing $(\mathrm{l})^{2}$ from $-\mathrm{L}$ to $+\mathrm{L}$, where $\mathrm{L}$ is an arbitrary maximum value of 1 , yield $\left\langle\mathrm{J}_{\mathrm{i}}^{2}\right\rangle=\sum_{\mathrm{l}_{\mathrm{i}}-\mathrm{L}}^{\mathrm{l}_{\mathrm{i}}=\mathrm{L}}(\hbar \mathrm{l})^{2} /(2 \mathrm{~L}+1)$ i.e., $\mathrm{J}^{2}=\sum_{\mathrm{i}=1}^{3}<\mathrm{J}_{\mathrm{i}}^{2}>=\mathrm{L}(\mathrm{L}+1) \hbar^{2}$.

The mere physical definition of angular momentum has been exploited to find quantum results completely analogous to that of the wave mechanics without any hypothesis on the details about the classical angular motion, actual nature of the particles and interaction potential to which is linked the angular momentum. Hence the Equation (5) and this last result yield Equation (6):

$$
\begin{aligned}
& \Delta \varepsilon=\varepsilon_{\text {ang }}-\varepsilon_{0} \quad \varepsilon_{\mathrm{G}}=-\varepsilon_{0}=\frac{\alpha_{1}}{2 \Delta \mathrm{r}} \\
& \varepsilon_{\text {ang }}=\varepsilon_{0} \frac{1(1+1) \hbar^{2}+2\left(\alpha_{2}-\mathrm{b}\right) \mu}{(\mathrm{n} \hbar)^{2}+2 \mathrm{~b} \mu}
\end{aligned}
$$

The binding energy is clearly $\varepsilon_{\mathrm{G}}=-\varepsilon_{\mathrm{o}}$. To assess this result, let us specify $\alpha_{1}$ putting first $\alpha_{2}=0$ and $b=0$ for simplicity, i.e., considering the first order term of the potential energy only; so $\varepsilon_{\mathrm{o}}$ and $\varepsilon_{\text {ang }}$ defining the range $\Delta \varepsilon$ refer to the potential energy $\varphi$ approximated by $\alpha_{1} / \Delta$ r only.

Examine first $\alpha_{1}=-Z^{2}$, as already inferred in (Tosto, $2013 \mathrm{~b}$ ); in this particular case $\varepsilon_{\mathrm{o}}=\mathrm{Z}^{2} \mathrm{e}^{4} \mu / 2(\mathrm{n \hbar})^{2}$, whereas the binding energy is $\varepsilon_{\mathrm{G}}=-\varepsilon_{\mathrm{o}}$ and $\Delta \mathrm{r}=(\mathrm{n} \hbar)^{2} / \mathrm{Ze}^{2} \mu$. One finds thus the old Bohr radius and the electron energy levels of the hydrogenlike atoms and ions, together with the well known condition $0 \leq 1 \leq n-1$ consistent with a bound state of the system. Under this condition only, indeed, $\Delta \varepsilon<0$; the negative sign means that the uncertainty range includes binding energy eigenvalues. This result has no specific interest for the purposes of the present paper, it has been quoted here as a formal check only. It is known that the quantum numbers $n$ and 1 resulting in the solution of the Schrodinger wave equation can take any values; this remind helps better understanding the postulated arbitrariness of the number of states in Equation (1), which in fact coincide with the respective quantum numbers.

More interesting and far reaching is instead the case where $\alpha_{1}=-\mathrm{G} \mu \mathrm{M}$ according to the Newton law, also inferred in (Tosto, 2013b); putting $\alpha_{2}=b=0$, one finds now that Equation (7):

$$
\begin{aligned}
\Delta r & =\frac{(\mathrm{n} \hbar)^{2}}{\mathrm{G} \mu^{2} \mathrm{M}} \quad \Delta \mathrm{P}_{\mathrm{r}}=\frac{\mathrm{G} \mu^{2} \mathrm{M}}{\mathrm{n} \hbar} \\
\varepsilon_{\mathrm{G}} & =-\frac{\mathrm{G}^{2} \mu^{3} \mathrm{M}^{2}}{2(\mathrm{n} \hbar)^{2}}=-\frac{\mathrm{G} \mu \mathrm{M}}{2 \Delta \mathrm{r}}
\end{aligned}
$$

are the first order eigenvalues of the quantized gravitational system; in particular $\varepsilon_{\mathrm{G}}$ is its binding energy. Moreover one also finds Equation (8):

$\varepsilon_{\text {ang }}=\left|\varepsilon_{\mathrm{G}}\right| \frac{1(1+1)}{\mathrm{n}^{2}} \quad \varepsilon_{0}=\left|\varepsilon_{\mathrm{G}}\right|$

The formal analogy with the Coulomb systems is due to that of the common kind of central potential $-\xi / \Delta \mathrm{r}$. In particular is still true that the bound state of energy $\varepsilon_{\mathrm{G}}$, i.e., the existence of an orbiting system itself, requires $0 \leq \mathrm{l} \leq \mathrm{n}-1$ at the Newtonian order of approximation. In conclusion, the third equality emphasizes that $\varepsilon_{\mathrm{G}}$ coincides with the potential energy of a reduced mass $\mu$ 
delocalized within a space uncertainty range $2 \Delta \mathrm{r}$ around the gravitational centre, in agreement with the physical meaning of reduced mass. The factor 2 is due to the fact that each eigenvalue $\varepsilon_{\mathrm{G}}$ is defined by the diametric uncertainty range $2 \Delta \mathrm{r}$ of $\mu$, as it is reasonable to expect, whereas the initial energy equation derived from the classical Equation (3) is defined as a function of $\Delta r$. Actually this result emphasizes that, as previously explained, the eigenvalues $\varepsilon_{\mathrm{G}}$ do not need specifying any particular reference system; $\mu$ is indeed defined for two masses orbiting around an orbital centre, whose position is however no longer specifiable and inessential.

It is instructive to conclude this section estimating $\mathrm{n}$ in the case of the system sun/earth, whose masses are about $2 \times 10^{30} \mathrm{Kg}$ and $6 \times 10^{24} \mathrm{Kg}$ respectively; their average distance $\Delta \mathrm{r}$ is about $1.5 \times 10^{11} \mathrm{~m}$. So the Equation (7) yield Equation (9):

$$
\begin{aligned}
& \mathrm{n}=\frac{\mu}{\hbar} \sqrt{\mathrm{GM} \Delta \mathrm{r}} \approx 10^{74} \quad \varepsilon_{\mathrm{G}}=-2.7 \times 10^{33} \mathrm{~J} \\
& \omega=\sqrt{\mathrm{MG} / \Delta \mathrm{r}^{3}}=2 \times 10^{-7} \mathrm{~s}^{-1}
\end{aligned}
$$

The last equation is justified soon below. It is known that an excited atom decays from its current $n$-dependent electron energy level to the ground state with $n=1$; it is thus reasonable to expect that the same holds also for the gravitational system. This allows defining the change $\delta \varepsilon_{\mathrm{G}}$ of $\varepsilon_{\mathrm{G}}$ of Equation (7) due to the change $\delta$ n during $\delta \Delta \mathrm{t}$; note in this respect that for changes $\delta \mathrm{n}$ of $\mathrm{n}$ as a function of time such that $\delta n<n$ it is possible to regard $\delta$ as a continuous variable.

Despite the Equation (7) and (15) do not depend explicitly upon the time, they nevertheless prospect the possibility of introducing via the change $\delta$ both $\delta \Delta \varepsilon_{\mathrm{G}} / \delta \Delta \mathrm{t}$ and $\delta \Delta \mathrm{r} / \delta \Delta \mathrm{t}$ : Indeed $\delta \mathrm{n}$ entails the change $\delta \Delta \varepsilon_{\mathrm{G}}$ of the initial range of eigenvalues as a function of $\delta \Delta \mathrm{t}$. As from now on is of interest the change of energy related to that of $\mathrm{n}$, which is balanced by a corresponding energy released as gravitational waves, let $\Delta \varepsilon_{\mathrm{G}}$ be the energy range including all eigenvalues $\varepsilon_{\mathrm{G}}=\varepsilon_{\mathrm{G}}(\mu, \mathrm{n}, \omega, \Delta \mathrm{r})$ with $\mathrm{n}$ falling between arbitrary $n_{o}$ and $n_{0} \pm \delta$; the notation $\Delta \varepsilon_{G}$ means that we are considering a range of values of $\varepsilon_{\mathrm{G}}$ corresponding to various values of $\mathrm{n}$, rather than any specific value of $\varepsilon_{\mathrm{G}}$ pertinent to its related value of $n$.

\section{QUANTUM MODEL OF GRAVITATIONAL WAVES}

This section considers for simplicity the Equation (7) instead of the more complete Equation (6); the physical meaning of the second order term of potential energy $\varphi$, here represented by the series coefficient $b$, will be concerned in the next section. Note that in Equation (7) appears the quantity MG, which has physical dimensions time $^{-2} \times$ length $^{3}$; this suggests that MG should be proportional to square frequency and cubic distance. In effect, assuming the following link between revolving frequency and orbital radius Equation (10):

$\omega^{2} \Delta \mathrm{r}^{3}=\mathrm{MG}$

It is possible to write Equation (11):

$\varepsilon_{\mathrm{G}} \Delta \mathrm{r}=-\frac{\mathrm{G} \mu \mathrm{M}}{2}=-\frac{\mu \mathrm{v}^{2}}{2} \Delta \mathrm{r} \quad \mathrm{v}=\omega \Delta \mathrm{r} \quad \omega=\frac{2 \pi}{\Delta \mathrm{r}}$

The well known position (10) is therefore justified without need of proportionality factor: As such, indeed, it gives $\varepsilon_{\mathrm{G}}$ the further physical meaning of link between the kinetic and potential energies of $\mu$ via the Equation (7). In effect one finds Equation (12):

$\overline{\mathrm{U}}=-\frac{\mathrm{G} \mu \mathrm{M}}{\Delta \mathrm{r}}=-\mu(\omega \Delta \mathrm{r})^{2}=-\mu \mathrm{v}^{2}$

which is nothing else but the virial theorem $\bar{U}=-2 \bar{T}$ valid also in quantum mechanics and already found itself in (Tosto, 2013b). Moreover, taking both signs allowed for $\sqrt{\omega^{2}}$ the Equation (10) also yields Equation (13):

$\varepsilon_{\mathrm{G}}= \pm \frac{\mathrm{n} \hbar \omega}{2}$

With the help of the Equation (7), which also reads $\mathrm{n} \hbar \omega= \pm\left(\varepsilon_{\mathrm{G}}-\varepsilon_{\mathrm{o}}\right)$; this expression is formally similar to that of the electromagnetic waves emitted/absorbed via electron transitions, indeed the double sign of $\delta\left(\varepsilon_{\mathrm{G}}-\varepsilon_{\mathrm{o}}\right) / \delta \mathrm{n}$ replaces the energy lost/gained by the atomic system.

Calculate now $\delta \bar{U} / \delta \Delta \mathrm{r}$, to which corresponds the radial force $-\mathrm{F}_{\mathrm{r}}$; since this force corresponds to the time change of radial momentum, then it must be true that $\Delta \dot{\mathrm{p}}_{\mathrm{r}}=\mathrm{F}_{\mathrm{r}}$, i.e., $\delta \overline{\mathrm{U}} / \delta \Delta \mathrm{r}=-\Delta \dot{\mathrm{p}}_{\mathrm{r}}$. This result is important as it introduces the time into the model despite all equations so far introduced do not depend explicitly upon $\Delta \mathrm{t}$. This is possible once admitting that $\Delta \mathrm{r}$ is allowed changing via $\mathrm{n}$ as a function of time, which is indeed reasonable as the Equation (1) link in principle all uncertainty range sizes to $\Delta \mathrm{t}$.

In fact, the uncertainty compels introducing itself the time together with the space dynamical variables.

To exploit these results note that the first equality of the Equation (12) yields $\delta \overline{\mathrm{U}} / \delta \Delta \mathrm{r}=\mathrm{G} \mu \mathrm{M} / \Delta \mathrm{r}^{2}$ and thus 
$\Delta \dot{\mathrm{p}}_{\mathrm{r}}=-\mu \omega^{2} \Delta \mathrm{r}$ describes a centripetal force $\mathrm{F}_{\mathrm{r}}^{\prime}=-\mu \omega^{2} \Delta \mathrm{r}$; the second equality yields instead $\delta \overline{\mathrm{U}} / \delta \Delta \mathrm{r}=-2 \mu \omega^{2} \Delta \mathrm{r}$, which describes thus a centrifugal force $F_{r}^{\prime \prime}=2 \mu \omega^{2} \Delta r$. This is because actually the former result concerns directly $\Delta r$ only, the latter $\omega \Delta r$ while being $\omega=\omega(\Delta r)$ because of Equation (10). The apparent ambiguity is therefore due to the way of regarding the variation $\delta \Delta \mathrm{r}$, which is not uniquely defined in the present model; as the Equation (1) relate $\Delta \mathrm{r}$ to $\Delta \mathrm{p}_{\mathrm{r}}$, the link between $\delta \Delta \mathrm{r}$ and $\delta \Delta \mathrm{p}_{\mathrm{r}}$ depends on how is regarded the number $n$ of states. Write in general:

$$
\delta \Delta \mathrm{p}_{\mathrm{r}}=\frac{\hbar \delta \mathrm{n}}{\Delta \mathrm{r}}-\frac{\mathrm{n} \hbar \delta \Delta \mathrm{r}}{\Delta \mathrm{r}^{2}}
$$

If $\mathrm{n}$ is constant, then Equation (14) yields $-\mu \omega^{2} \Delta \mathrm{r}=-$ $n \hbar \Delta \mathrm{r}^{-2} \delta \Delta \mathrm{r} / \delta \Delta \mathrm{t}$ i.e., $\delta \Delta \mathrm{r} / \delta \Delta \mathrm{t}=\mu \omega^{2} \Delta \mathrm{r}^{3} / \mathrm{n} \hbar$; the relationship of $\delta \Delta \mathrm{p}_{\mathrm{r}} / \delta \Delta \mathrm{t}$ with $\mathrm{F}_{\mathrm{r}}^{\prime}$ has been guessed on the basis of their common minus sign. Moreover according to the Equation (10) the last equation reads $\mu \mathrm{MG} / \mathrm{n} \hbar=\delta \Delta \mathrm{r} / \delta \Delta \mathrm{t}$ and thus, thanks to the second Equation (7), $\delta \Delta r / \delta \Delta t=v_{r}$ being $v_{r}=$ $\Delta \mathrm{p}_{\mathrm{r}} / \mu$; making explicit $\Delta \mathrm{p}_{\mathrm{r}}=\mathrm{p}_{\mathrm{r} 2}-\mathrm{p}_{\mathrm{r} 1}$, therefore, the net radial deformation rate of $\Delta \mathrm{r}$ results related to that of the boundaries $\mathrm{p}_{\mathrm{r} 2} / \mu$ and $\mathrm{p}_{\mathrm{r} 1} / \mu$ of the radial momentum range.

With n not constant, instead, Equation (7) yields $\delta \Delta \mathrm{r}$ $=2 \Delta \mathrm{r} \delta \mathrm{n} / \mathrm{n}$; replacing into the Equation (14) one finds $\delta \Delta \mathrm{p}_{\mathrm{r}}=-\mathrm{n} \hbar \delta \Delta \mathrm{r} / 2 \Delta \mathrm{r}^{2}$.

These equations emphasize how to define the time derivatives $\Delta \dot{\mathrm{p}}_{\mathrm{r}}$ of the radial momentum uncertainty range. Of course is of interest here just the case of variable $n$ to infer the sought equation of the gravitational waves; hence, equating $\delta \Delta \mathrm{p}_{\mathrm{r}} / \delta \Delta \mathrm{t}$ to $\mathrm{F}_{r}^{\prime \prime}$, one finds Equation (15):

$$
\frac{\delta \Delta \mathrm{p}_{\mathrm{r}}}{\delta \Delta \mathrm{t}}=2 \mu \omega^{2} \Delta \mathrm{r} \quad \frac{\delta \Delta \mathrm{r}}{\delta \Delta \mathrm{t}}=-\frac{4 \mu \mathrm{MG}}{\mathrm{n} \hbar}
$$

Consider now two constant quantities that characterize the system orbiting circularly, i.e., $v=\omega \Delta r$ and $\omega^{2} \Delta r^{3}=$ $\mathrm{MG}$; these equations are exploited to find the relationship between $\delta \omega$ and $\delta \Delta \mathrm{r}$ as a function of $\delta \mathrm{n}$, which indeed is the quantity expected to change as a function of $\delta \Delta \mathrm{t}$. One finds $\Delta r^{3} \delta\left(\omega^{2}\right)+3 \omega^{2} \Delta r^{2} \delta \Delta r=0$, whereas the first Equation (7) yields also $\delta \Delta \mathrm{r}=(2 \Delta \mathrm{r} / \mathrm{n}) \delta \mathrm{n}$; hence $\delta\left(\omega^{2}\right)+6 \omega^{2} \delta \mathrm{n} / \mathrm{n}=$ 0 yields $\omega^{2}=-n \delta\left(\omega^{2}\right) / 6 \delta$ n and thus Equation (16):

$\mathrm{n} \hbar \omega^{2}=-\frac{\mathrm{n}^{2} \hbar \delta\left(\omega^{2}\right)}{6 \delta \mathrm{n}} \quad \delta \mathrm{n} / \delta\left(\omega^{2}\right)<0$

The inequality means that when decreasing $\mathrm{n}$ must increase $\omega$, as it is reasonable to expect recalling that $n$ controls the extent of $\Delta \mathrm{r}$ : The closer is $\mu$ to the orbit centre, the faster must be its revolution rate. Consider now that the variation $\delta \mathrm{n}$ is an arbitrary integer, in principle not dependent on the initial $\mathrm{n}$ and having sign opposite to that of $\delta\left(\omega^{2}\right)$; this agrees with the fact that gravitational waves are emitted when $n$ decreases down to its ground value $n=$ 1 during the decay of the orbiting energy, whereas conversely they excite the orbiting system if absorbed. Clearly, even $\delta \omega$ does not depend on the initial $\omega$. As the last equation has the form $3 \omega^{2} \delta\left(n^{2}\right)=-n^{2} \delta\left(\omega^{2}\right)$ it seems reasonable to guess that $\omega^{2} \delta\left(n^{2}\right)=$ const so that $n^{2} \delta\left(\omega^{2}\right)=-$ 3 const as well; this confirms the opposite signs of $\delta\left(\mathrm{n}^{2}\right)$ and $\delta\left(\omega^{2}\right)$. Then it also must be true that $n \hbar \omega^{2}=\hbar \operatorname{cost} / 2 \delta n$; moreover by dimensional reasons it seems rational to think const proportional to the square Planck frequency $\omega_{\mathrm{pl}}^{2}$ via an appropriate proportionality factor $\mathrm{q}$. Thus one obtains:

$\mathrm{n} \hbar \omega^{2}=\frac{\mathrm{W}_{\mathrm{pl}}}{\mathrm{q} \delta \mathrm{n}} \quad \mathrm{W}_{\mathrm{pl}}=\hbar \omega_{\mathrm{pl}}^{2}=\frac{\mathrm{c}^{5}}{\mathrm{G}}$

being $\mathrm{W}_{\mathrm{pl}}$ the Planck power. Therefore it is possible to write the following chain of equations with the help of the Equation (17) and (15) Equation (18):

$\frac{\delta \Delta \mathrm{r}}{\delta \Delta \mathrm{t}}=-4 \frac{\mu \omega \mathrm{MG}}{\mathrm{n} \hbar \omega}=-4 \mathrm{q} \delta \mathrm{n} \frac{\mu \omega^{2} \mathrm{MG}}{\mathrm{W}_{\mathrm{pl}}}$

An analogous procedure is now carried out for $\Delta \varepsilon_{\mathrm{G}}$ with the help of this last result; owing to the last equation (7), the chain of equations is now Equation (19):

$$
\begin{aligned}
& \frac{\delta \Delta \varepsilon_{\mathrm{G}}}{\delta \Delta \mathrm{t}}=\frac{\delta \Delta \varepsilon_{\mathrm{G}}}{\delta \Delta \mathrm{r}} \frac{\delta \Delta \mathrm{r}}{\delta \Delta \mathrm{t}}= \\
& -2 \mathrm{q} \delta \mathrm{n} \frac{(\mathrm{M} \mathrm{\mu \textrm {G }})^{2} \omega^{6}}{\Delta \mathrm{r}^{2} \mathrm{~W}_{\mathrm{pl}}}=-2 \mathrm{q} \delta \mathrm{n} \frac{\mu^{2} \omega^{6} \Delta \mathrm{r}^{4}}{\mathrm{~W}_{\mathrm{pl}}}
\end{aligned}
$$

Note that this last result reads also:

$$
\frac{\delta \Delta \varepsilon_{\mathrm{G}}}{\delta \Delta \mathrm{t}}=4 \mathrm{q} \delta \mathrm{n} \frac{(\mathrm{n} \hbar \omega)^{2} \Delta \varepsilon_{\mathrm{G}}}{\mu \Delta \mathrm{r}^{2} \mathrm{~W}_{\mathrm{pl}}}
$$

which also introduces $v_{\mathrm{gw}}=\left(\delta \Delta \varepsilon_{\mathrm{G}} / \Delta \varepsilon_{\mathrm{G}}\right) / \delta \Delta \mathrm{t}$, being $v_{\mathrm{gw}}$ the frequency corresponding to the energy loss $\delta \Delta \varepsilon_{\mathrm{G}} / \Delta \varepsilon_{\mathrm{G}}$ per unit time. To make understandable the physical meaning of this result write $2(n \hbar \omega)^{2} / \mu \Delta r^{2}=\left(n \hbar \omega^{2}\right)^{2} / \bar{T}$, whose right hand side reduces with the help of the Equation (17) and (12) to $\mathrm{W}_{\mathrm{pl}}^{2} /\left(\mathrm{q}^{2} \overline{\mathrm{T}} \delta \mathrm{n}^{2}\right)$. Hence, replacing into the Equation (20), one obtains $2 \mathrm{qv}_{\mathrm{gw}} \delta \mathrm{n}=\mathrm{W}_{\mathrm{pl}} / \overline{\mathrm{T}}$ and thus Equation (21): 
$\omega_{\mathrm{gw}} \delta \mathrm{n}=\frac{\mathrm{W}_{\mathrm{pl}}}{\overline{\mathrm{T}}} \quad \omega_{\mathrm{gw}}=2 \pi v_{\mathrm{gw}} \quad \mathrm{q}=\pi$

With the last position, the frequency of the wave irradiated appears expressed at left hand side per revolution period, coherently with $\omega$ defining $\overline{\mathrm{T}}$. The smaller this latter, the greater the frequency of the wave irradiated; being $\overline{\mathrm{T}}$ the current kinetic energy of $\mu$ in the orbiting system dissipating energy, its small residual value indicates great energy lost, in agreement with the inequality (16). The Planck power plays here the role of proportionality constant. Moreover it is interesting the chance of expressing $v_{\mathrm{gw}}$ as a function of the Planck frequency $v_{\mathrm{PI}}$ with the given definition of q Equation (22):

$h v_{\mathrm{gw}} \delta \mathrm{n}=\frac{\left(\mathrm{h} v_{\mathrm{pl}}\right)^{2}}{\overline{\mathrm{T}}}$

Eventually, replacing q into Equation (18) and (19), one finds:

$$
\begin{aligned}
& \left(\frac{\delta \Delta \varepsilon}{\delta \Delta \mathrm{t}}\right)_{\text {quant }}=-2 \pi \frac{\mathrm{G} \mu^{2} \Delta \mathrm{r}^{4} \omega^{6}}{\mathrm{c}^{5}} \delta \mathrm{n} \\
& \left(\frac{\delta \varepsilon}{\delta \mathrm{t}}\right)_{\text {Einst }}=-\frac{32}{5} \frac{\mathrm{G} \mu^{2} \Delta \mathrm{r}^{4} \omega^{6}}{\mathrm{c}^{5}} \\
& \left(\frac{\delta \Delta \varepsilon}{\delta \Delta \mathrm{t}}\right)_{\text {quant }}=-4 \pi \frac{\mathrm{G} \mu \Delta \mathrm{r}^{3} \omega^{4}}{\mathrm{c}^{5}} \delta \mathrm{n} \\
& \left(\frac{\delta \mathrm{r}}{\delta \mathrm{t}}\right)_{\text {Einst }}=-\frac{64}{5} \frac{\mathrm{G} \mu \Delta \mathrm{r}^{3} \omega^{4}}{\mathrm{c}^{5}}
\end{aligned}
$$

The second set reports the well known Einstein equation of energy loss of a gravitational system via emission of gravitational waves. It appears that the functional dependence of the energy loss on the quantities that characterize the circular orbiting motion of the reduced mass $\mu$ is identical to that of the quantum formulas; however, the constant factors $32 / 5$ and $64 / 5$ of the relativistic formulas are replaced by $2 \pi \delta$ n and $4 \pi \delta n$ of the respective quantum results. Note that the relativistic numerical factors differ from the respective $2 \pi$ and $4 \pi$ by less than $2 \%$, which shows that the present result substantially agrees with that of Einstein for $\delta \mathrm{n}=$ 1 ; in effect the change of the integer $\mathrm{n}$ can be nothing else but any integer itself. However just the presence of $\delta \mathrm{n}$ is the main difference, peculiar of the present approach. Note that the differential $\delta \Delta t=\delta\left(t-t_{0}\right)$ reduces to the differential $\delta t$ if the lower boundary $t_{o}$ of the time uncertainty range is constant; the same holds also for $\delta \Delta \varepsilon$ $=\delta\left(\varepsilon-\varepsilon_{0}\right)$, which reduces to $\delta \varepsilon$ if in particular is constant $\varepsilon_{0}$. Moreover, as in fact the energy loss is due to the emission of gravitational waves via transitions between gravitational energy levels of the orbiting system, it appears natural to expect that this loss should contain an integer factor accounting for various higher frequencies of the waves; so $\delta \Delta \varepsilon$ must be intended as alteration of the range of energy levels per unit gap of gravitational quantum number per unit change of time range. Otherwise stated more shortly: $\delta \Delta \varepsilon$ is due to the deformation of the space-time, $\delta \Delta \mathrm{r}$ and $\delta \Delta \mathrm{t}$, in the presence of an orbiting system. Once again, replacing local values with uncertainty ranges is the key point of the present model: As any expression having the form $\chi \delta$ with $\chi=\chi(\omega, \Delta \mathrm{r})$ and $\delta \mathrm{n}=\mathrm{n}_{2}-\mathrm{n}_{1}$ reads actually $\Delta \chi=$ $\chi \mathrm{n}_{2}-\chi \mathrm{n}_{1}$, it follows that the left hand sides of the quantum Equation (23) and (24) are quantized changes included in the range $\Delta \chi$ defined by $\mathrm{n}_{1}<\mathrm{n}<\mathrm{n}_{2}$.

Eventually, changing the sign of $\delta$ means that $\delta \Delta \varepsilon_{\mathrm{G}} / \delta \Delta \mathrm{t}$ and $\delta \Delta \mathrm{r} / \delta \Delta \mathrm{t}$ change sign as well; i.e., the gravitational system gains energy when increasing its number of allowed states. In other words, the fact that either sign is allowed to $\delta \mathrm{n}$ shows that emission and absorption of gravitational waves are symmetric processes. Owing to the fact that $n$ of orbiting systems is very high, i.e., it can be regarded in practice as a continuous variable, it is reasonable to expect that different gravitational systems can exchange energy via gravitational waves.

\section{THE BLACK HOLE EFFECT}

Consider a hypersphere of radius $\Delta \mathrm{r}$ enclosing the total mass $\mathrm{M}$ of the orbiting system; the average density within this hypersphere is $\rho=3 \mathrm{M} / 4 \pi \Delta \mathrm{r}^{3}$, which also yields the energy density per unit area $\rho c^{2} \Delta \mathrm{r} / 3=$ $\mathrm{Mc}^{2} / 4 \pi \Delta \mathrm{r}^{2}$ at the surface of the hypersphere. Moreover also MG $\rho$ has physical dimensions of energy per unit surface, as indeed it is proportional to $\mathrm{M \omega}^{2}$; multiplying thus this latter by $\omega$ one finds the related power per unit surface as well. This section implements the chance of calculating bulk and surface energy densities via $\rho$. The particular case, in principle possible, where $\mathrm{M}$ and $\Delta \mathrm{r}$ fulfil the black hole condition found in (Tosto, 2013b) $\Delta \mathrm{r}_{\mathrm{bh}}$ $=2 \mathrm{MG} / \mathrm{c}^{2}$ is concerned below; the subscript bh stands for black hole from now on. In effect nothing excludes in principle the possibility that, given a hypersphere of radius $\Delta \mathrm{r}_{\mathrm{bh}}$ enclosing M, is verified the condition Equation (25):

$\rho_{\mathrm{bh}}=\frac{3 \mathrm{M}}{4 \pi \Delta \mathrm{r}_{\mathrm{bh}}^{3}}=\frac{3 \mathrm{c}^{6}}{32 \pi \mathrm{G}(\mathrm{MG})^{2}}$ 
Replacing $\Delta \mathrm{r}_{\mathrm{bh}}$ into the Equation (7) one finds $\varepsilon_{\mathrm{bh}}=$ $\mu \mathrm{c}^{2} / 4$, to which corresponds the maximum value of angular momentum $\mu \mathrm{c}^{2} / 4=\mathrm{J}_{\mathrm{bh}}^{2} / 2 \mu \Delta \mathrm{r}_{\mathrm{bh}}^{2}$ still consistent with a bound state; $\mathrm{J}_{\mathrm{bh}}^{2}=\left(\mu \mathrm{c} \Delta \mathrm{r}_{\mathrm{bh}} / 2\right)^{2}$ yields then $\left|\mathbf{J}_{\mathrm{bh}}\right|=$ $\mathrm{m}_{1} \mathrm{~m}_{2} \mathrm{G} / \mathrm{c}$, which reads in particular $\left|\mathbf{J}_{\mathrm{bh}}\right|=\mathrm{m}^{2} \mathrm{G} / \mathrm{c}$ for $\mathrm{m}_{1}$ $=\mathrm{m}_{2}=\mathrm{m}$. Moreover at the event horizon of the mass $\mathrm{M}$ the equation (25) yields the energy $\rho_{\mathrm{bh}} \mathrm{c}^{2} \Delta \mathrm{r}_{\mathrm{bh}} / 3=\mathrm{Mc}^{2} / 4 \pi \Delta \mathrm{r}_{\mathrm{bh}}^{2}$ per unit surface, which can be also expressed as a function of $\rho_{\mathrm{bh}} / 3$ as follows:

$$
\frac{\mathrm{MG} \rho_{\mathrm{bh}}}{3}=\frac{2}{\Delta \mathrm{r}_{\mathrm{bh}}} \frac{\mathrm{c}^{4}}{32 \pi \mathrm{G}}
$$

The papers (Tosto, 2012; 2013b) have shown the physical meaning of event horizon at $\Delta r=\Delta r_{b h}$ in the frame of the quantum approach based on the Equation (1); this fact encourages therefore extending the results of the previous section also to the particular case where $\mathrm{M}$ and $\Delta \mathrm{r}$ are effectively subjected to the constrain of the Equation (25), according which the orbit plane becomes the diametric section of a hypersphere of radius $\Delta \mathrm{r}_{\mathrm{bh}}$. In principle one could suspect that no emission of gravitational waves should be allowed in such a case; indeed the gravitational waves subtract energy to the system, which therefore tends to shrink its size. However transferring any kind of radiation energy away from the event horizon of the black hole identified by such a hypersphere should be forbidden, whence the question: Is it possible to confirm such a conclusion in the frame of the present quantum model? In practice the impossibility of energy radiation could occur by forming a gravitational wave that however is trapped at the event horizon and falls back into the orbiting system, thus releasing to this latter its initial formation energy; as the global energy balance of such a process is null, one concludes that the effective release of gravitational waves is hindered. Assume therefore that the left hand side of the Equation (26) represents an energy density that cannot be irradiated. This equation is however useful to assess approximately the energy per unit surface at a distance very close to and in fact just beyond the event horizon, which therefore can be irradiated outwards as a gravitational wave. So, before concerning the actual black body condition, let us try to estimate the energy of the wave emitted as a function of that stored near the black hole boundary; to this purpose we implement an energy expectedly similar to that calculated just at the right hand side of the Equation (26) via the known expression of $\Delta \mathrm{r}_{\mathrm{bh}}$. Express thus the energy per unit surface at any $\Delta \mathrm{r}>\Delta \mathrm{r}_{\mathrm{bh}}$ also as $\overline{\mathrm{U}} / \mathrm{A}=\mu\left(\omega_{\mathrm{bh}} \Delta \mathrm{r}_{\mathrm{bh}}\right)^{2} / \mathrm{A}$ via the surface $A$ to be defined; $\bar{U}$ is regarded in the same way, i.e., it correlates the wave energy emissible to the potential energy of $\mu$ just outside the event horizon. Linking the Equation (26) to this latter with a proportionality constant $\xi$, one finds:

$$
\frac{\mu\left(\omega_{\approx \mathrm{bh}} \Delta \mathrm{r}_{\approx \mathrm{bh}}\right)^{2}}{\mathrm{~A}}=\xi \frac{2}{\Delta \mathrm{r}_{\approx \mathrm{bh}}} \frac{\mathrm{c}^{4}}{32 \pi \mathrm{G}}
$$

with notation $\approx$ bh emphasizing that $\omega$ and $\Delta r$ of the system are close to that of the Equation (26). This yields:

$$
\xi=\frac{\Delta \mathrm{r}_{\approx \mathrm{bh}}}{2 \mathrm{~A}} \frac{32 \pi \mathrm{G} \mu \mathrm{v}^{2}}{\mathrm{c}^{4}} \quad \mathrm{v}^{2}=\left(\omega_{\approx \mathrm{bh}} \Delta \mathrm{r}_{\approx \mathrm{bh}}\right)^{2}
$$

To guess the appropriate definition of $\xi$ and A, consider the emission of waves perpendicularly to the plane of the orbit. Define therefore the area A as shown in the Fig. 1, i.e., in order to introduce an observation point of the transiting wave at the distance $\Delta r$ from the boundary surface, which is a distance $\Delta \mathrm{r}_{\mathrm{bh}}$ apart from the orbit centre; the observer is at the vertex of an ideal cone whose base is the orbit area $\pi \Delta \mathrm{r}_{\approx \mathrm{bh}}^{2}$. The measure apparatus collects the waves coming from the orbit plane and included within the lateral surface $\mathrm{A}=\pi \Delta \mathrm{r}_{\approx \mathrm{bh}} \sqrt{\Delta \mathrm{r}_{\approx \mathrm{bh}}^{2}+\left(\Delta \mathrm{r}+\Delta \mathrm{r}_{\approx \mathrm{bh}}\right)^{2}}$ of the cone, as it is shown in Fig. 2. So $A \approx \pi \Delta r_{\approx b h} \Delta r$, if $\Delta r>>\Delta r_{\approx b h}$; whence $\Delta \mathrm{r}_{\approx \mathrm{bh}} / 2 \mathrm{~A} \approx(2 \pi \Delta \mathrm{r})^{-1}$. Hence:

$$
\xi=\frac{16 \mathrm{G} \mu \mathrm{v}^{2}}{\mathrm{c}^{4} \Delta \mathrm{r}}
$$

So, aside from the numerical coefficient, $\xi$ defined above results to be the ratio between the available potential energy of $\mu$ and the Planck force times the propagation distance of the wave to the detection point; i.e., $\xi \propto \eta\left(\Delta r_{b h}\right) / \eta(\Delta r)$, where $\eta$ stands for energy at the respective distances. The fact that $\xi \rightarrow 0$ for $\Delta \mathrm{r} \rightarrow \infty$ confirms that $\xi$ yields the attenuation of the local intensity of the gravitational wave at the distance $\Delta \mathrm{r}$. Note that in the particular case where $\mathrm{m}_{1}=\mathrm{m}_{2}=\mathrm{m}$ the last equation yields:

$$
\xi=\frac{8 \mathrm{Gmv}^{2}}{\mathrm{c}^{4} \Delta \mathrm{r}}
$$

i.e., the familiar Einstein's expression of total intensity of gravitational waves at a point $\Delta \mathrm{r}$ apart from the orbit centre. 


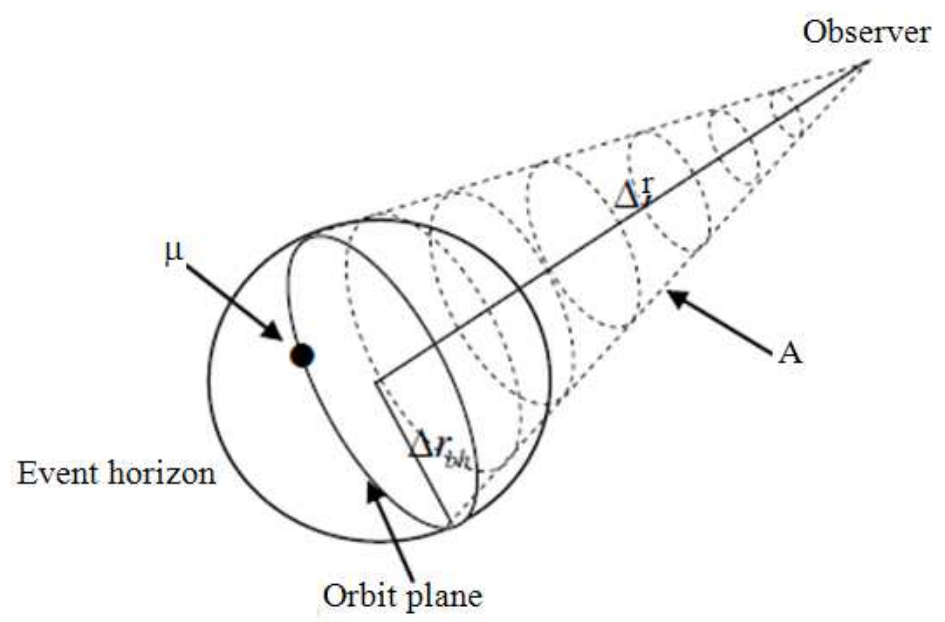

Fig. 1. The gravitational wave irradiated from the area $\pi \Delta \mathrm{r}_{\mathrm{bh}}^{2}$ of the orbital plane of the reduced mass $\mu$ is received by an observer at a distance $\Delta \mathrm{r}$

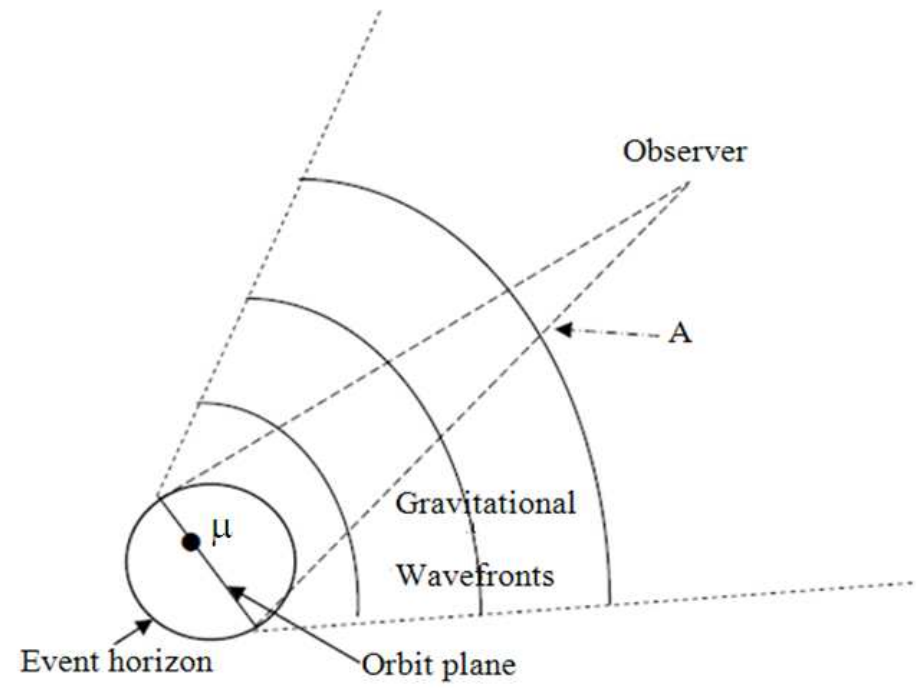

Fig. 2. The intensity of the gravitational wave is consistent with the aperture angle of the ideal cone of the Fig. 1, whose internal surface A intercepts the propagating wave front. The position of the observer defines the measure point at a distance $\Delta \mathrm{r}$ normal to the orbit plane

This reasoning also shows that the gravitational waves must be emitted at rate equal to c: Only an emission rate asymptotically tending to the speed of light could escape from an external point asymptotically close to the event horizon surface of the mass $\mathrm{M}$. This conclusion will be confirmed via additional and more fundamental considerations in the next section.

To show the black hole feature of the Equation (25) and verify the related assumption on the Equation (26) it is necessary to start from Equation (6) more general than the simpler Equation (7), which also gives way of explaining the physical meaning of $\Delta \mathrm{r}$ including the coefficient $b$. Note first of all that whatever the analytical expression of $(n \hbar)^{2}+2 b \mu$ might be, $\varepsilon_{o}$ of Equation (6) still reads $\left|\alpha_{1} / 2 \Delta \mathrm{r}\right|=\varepsilon_{\mathrm{o}}$ like in the Equation (7) and (8) due to the Equation (5). This conclusion is easily understood recalling that the boundary values of the uncertainty ranges are arbitrary, unknown and undeterminable in principle by fundamental assumption; so they cannot play any role in the present model. To show this point, 
note that $2 b \mu$ in the Equation (5) suggests writing (nћ) ${ }^{2}+2 b \mu$ $=(\mathrm{n} \hbar+\mathrm{b} \mu / \mathrm{n} \hbar)^{2}-(\mathrm{b} \mu / \mathrm{n \hbar})^{2}$; so rewrite Equation (27):

$\Delta \mathrm{r}=\mathrm{r}_{1}-\mathrm{r}_{0}=\frac{(\mathrm{n} \hbar+\mathrm{b} \mu / \mathrm{n} \hbar)^{2}}{\mu \alpha_{1}}-\frac{(\mathrm{b} \mu / \mathrm{n} \hbar)}{\mu \alpha_{1}}$

Whatever $\alpha_{1}$ might be; clearly these considerations hold in principle both for Coulomb and planetary systems. The fact that the addends at right hand side reproduce the range boundaries, requires that $(\mathrm{n} \hbar+\mathrm{b} \mu / \mathrm{nh})^{2}$ and $(\mathrm{b} \mu / \mathrm{nh})^{2}$ should have themselves an analogous form; in other words it appears reasonable to expect that the presence of $b$ does not contradict the first order form of $\Delta \mathrm{r}$, but it should simply broaden and generalize that of Equation (7). In this respect also note that Equation (28):

$\Delta \mathrm{r}^{\prime}=\mathrm{r}_{1}-\mathrm{r}_{0}^{\prime}=\frac{(\mathrm{n} \hbar+\mathrm{b} \mu / \mathrm{n} \hbar)^{2}}{\mu \alpha_{1}} \quad \mathrm{r}_{0}^{\prime}=\mathrm{r}_{0}+\frac{(\mathrm{b} \mu / \mathrm{n} \hbar)}{\mu \alpha_{1}}$

Yet, as previously stated, changing $r_{0}$ to $r_{0}^{\prime}$ means merely changing the position of the range with respect to the origin of the reference system $\mathrm{R}$ initially introduced or, identically, changing reference system. However all reference systems are physically indistinguishable and thus equivalent in calculating the observables via the uncertainty ranges of Equation (1) as previously shown for the angular momentum and the energy levels. In fact $\Delta \mathrm{r}^{\prime}$ is physically indistinguishable from $\Delta \mathrm{r}$; being $\mathrm{r}_{0}$ and $\mathrm{r}_{1}$ arbitrary, any value allowed for the latter is identically allowed for the former as well. In effect the section 2 has shown that neither $r_{1}$ and $r_{0}$ nor the corresponding boundary values of the radial momentum range need being specified to obtain the correct quantum eigenvalues of angular momentum and energy; rather, appears crucial their quantization condition via the Equation (1). So the lack of any hypothesis about the uncertainty ranges, in particular about their actual sizes, suggests regarding $b$ in order that:

$$
\begin{array}{ll}
\frac{\mathrm{b} \mu}{\mathrm{n} \hbar}=\mathrm{n}^{\prime} \hbar & \Delta \mathrm{r}=\frac{\left(\mathrm{n}^{\prime \prime} \hbar\right)^{2}}{\mu \alpha_{1}}-\frac{\left(\mathrm{n}^{\prime} \hbar\right)^{2}}{\mu \alpha_{1}} \\
\mathrm{n}^{\prime \prime}=\mathrm{n}+\mathrm{n}^{\prime} & \varepsilon_{0}=\frac{1}{2} \frac{\mu \alpha_{1}^{2}}{\left(\mathrm{n}^{\prime \prime} \hbar\right)^{2}-\left(\mathrm{n}^{\prime} \hbar\right)^{2}}
\end{array}
$$

being n' a further integer arbitrary itself like n; the fourth equation is the generalization at the second order of the potential of $\varepsilon_{\mathrm{o}}$ of the Equation (6), which is indeed obtained as a particular case for $n$ ' $=0$. Define thus Equation (29 and 30):

$$
\begin{aligned}
& \Delta \mathrm{r}_{\text {ell }}=\frac{\left(\mathrm{n}^{\prime \prime} \hbar\right)}{\mu \alpha_{1}} \quad \varepsilon_{\text {ell }}=-\frac{1}{2} \frac{\mu \alpha_{1}^{2}}{\left(\mathrm{n}^{\prime \prime} \hbar\right)^{2}} \\
& \Delta \mathrm{r}_{\text {circ }}=\frac{\left(\mathrm{n}^{\prime \prime} \hbar\right)-\left(\mathrm{n}^{\prime} \hbar\right)}{\mu \alpha_{1}} \quad \varepsilon_{\text {circ }}=-\frac{1}{2} \frac{\mu \alpha_{1}^{2}}{\left(\mathrm{n}^{\prime \prime} \hbar\right)^{2}-\left(\mathrm{n}^{\prime} \hbar\right)^{2}}
\end{aligned}
$$

The definitions are due to the fact that $\varepsilon_{\text {ell }}$ corresponds to $\Delta \mathrm{r}$ dependent on $\mathrm{n}^{2}$ and $\mathrm{n}^{\prime 2}$, whereas $\varepsilon_{\text {circ }}$ corresponds to $\Delta \mathrm{r}$ dependent on $\mathrm{n}^{2}$ and $\mathrm{n}$ ' in such a way however to remove its dependence upon $\mathrm{n}^{2}$; so the subscripts emphasize that in the former case only $\Delta \mathrm{r}$ corresponds to elliptic orbits, whereas in the latter case $\Delta \mathrm{r}$ still describes circular orbits in a different reference system. This clearly appears noting that classically Equation (31):

$$
\varepsilon_{\text {ell }} / \varepsilon_{\text {circ }}=1-\mathrm{e}^{2} \quad \mathrm{e}=\mathrm{n}^{\prime} /\left(\mathrm{n}+\mathrm{n}^{\prime}\right)<1
$$

as it is well known; this conclusion was already inferred in (Tosto, 2000). It is also clear that if $\alpha_{1}=-\mathrm{Ze}^{2}$ the Equation (7) correspond to the Bohr circular electron orbits, whereas instead $\varepsilon_{\text {ell }}$ corresponds to the elliptic orbits of the Sommerfeld model; although the quantum $\varepsilon_{\text {ell }}$ is formally indistinguishable from $\varepsilon_{\mathrm{G}}$ of Equation (7) because any value allowed to n" is also allowed to n, in agreement with the aforesaid requirement, remains however the fact that $\mathrm{n}$ " is determined by two independent values of quantum numbers rather than by one quantum number only. At the second order truncation of the interaction potential $\varphi$ the energies of Bohr and Sommerfeld electron orbits are degenerate, as it is known. Of course today the modern quantum mechanics overcomes this aged reference to the classical physics, justifiable at the time where the new born quantum mechanics was still gripped to the well established and long trusted classical physics. In effect the present quantum model does not need any phenomenological reference to the concept of orbit or trajectory; it has been shown that the mere physical definition of angular momentum leads to the quantized values without any hypothesis about the nature of the particle or its angular motion around the nucleus. Yet the concept of orbit is still useful when concerning the quantum behaviour of macroscopic objects: In effect the short remind to the hydrogenlike atoms has been once again sketched as a check of considerations actually aimed to describe planetary systems only. Let us return thus to the gravitational waves. Since both $n$ and $n$ ' depend on time only, indeed $\Delta \mathrm{r}$ and $\varepsilon_{\mathrm{G}}$ depend on the time just because of them, let us rewrite the 
Equation (18) in order to emphasize its dependence on $\delta \Delta \mathrm{t}$ via $\delta \mathrm{n}$ and $\delta \mathrm{n}$ ' as follows:

$$
\frac{\delta \Delta \mathrm{r}}{\delta \Delta \mathrm{t}}=\frac{\delta \Delta \mathrm{r}}{\delta \mathrm{n}} \frac{\delta \mathrm{n}}{\delta \Delta \mathrm{t}}+\frac{\delta \Delta \mathrm{r}}{\delta \mathrm{n}^{\prime}} \frac{\delta \mathrm{n}^{\prime}}{\delta \Delta \mathrm{t}}
$$

To specify the interest to describe the orbiting system at the event horizon, however, it is also necessary to impose the condition:

$$
\frac{\delta \Delta \mathrm{r}}{\delta \Delta \mathrm{t}}=\frac{\delta \mathrm{n}}{\delta \Delta \mathrm{t}}\left(\frac{\delta \Delta \mathrm{r}}{\delta \mathrm{n}}+\frac{\delta \Delta \mathrm{r}}{\delta \mathrm{n}^{\prime}} \frac{\delta \mathrm{n}^{\prime}}{\delta \Delta \mathrm{t}} \frac{\delta \Delta \mathrm{t}}{\delta \mathrm{n}}\right)=0
$$

still regarding for simplicity $\delta$ and $\delta n^{\prime}$ as differentials; in fact this equation hinders energy dissipation via gravitational waves concurrent to the shrinking of the orbit radius, see Equation (19). This case is coherent with that previously carried out in section 4 , as also now $\Delta \mathrm{r}$ given by Equation (30) is still pertinent to circular orbits despite the presence of n'. Intending therefore $\operatorname{circ} \Delta \mathrm{r} \equiv \Delta \mathrm{r}_{\text {circ }}$, calculate:

$$
\frac{\delta \Delta \mathrm{r}}{\delta \mathrm{n}}+\frac{\delta \Delta \mathrm{r}}{\delta \mathrm{n}^{\prime}} \frac{\delta \mathrm{n}^{\prime}}{\delta \mathrm{n}}=0
$$

That yields the equation $n^{\prime}+n \delta n^{\prime} / \delta n+n=0$ whose solution is $n^{\prime}=$ const $/ n-n / 2$. The integration constant is calculated replacing this solution into the equation of $\Delta \mathrm{r}_{\mathrm{bh}}$ $=2 \mathrm{MG} / \mathrm{c}^{2}$, which expresses the black hole condition for the total mass $\mathrm{M}$ of the gravitational system. Thus, owing to the Equation (7), $2 \hbar^{2}$ const $/\left(\mu^{2} \mathrm{MG}\right)=2 \mathrm{MG} / \mathrm{c}^{2}$ yields:

$$
\mathrm{n}^{\prime}=\mathrm{n}\left[\left(\frac{\mu \mathrm{MG}}{\mathrm{n} \hbar \mathrm{c}}\right)^{2}-\frac{1}{2}\right] \quad \frac{\mu \mathrm{MG}}{\mathrm{n} \hbar \mathrm{c}} \geq \frac{1}{\sqrt{2}} \quad \Delta \mathrm{r} \leq \Delta \mathrm{r}_{\mathrm{bh}}
$$

Which must fulfil the given condition because both $\mathrm{n}$ and $\mathrm{n}$ ' are positive. Being $\mathrm{n} \hbar=\sqrt{\mathrm{GM} \mu^{2} \Delta \mathrm{r}}$ is immediately inferred the second inequality in Equation (32), which holds for any $\mathrm{n}$ fulfilling the black hole condition. This conclusion is obvious: It expresses the fact that to form a black hole of mass $\mathrm{M}$, the size of $\Delta \mathrm{r}$ cannot overcome a critical value consistent with that allowed by $\mathrm{MG}=\omega_{\mathrm{bh}}^{2} \Delta \mathrm{r}_{\mathrm{bh}}^{3}$, which is clearly controlled by $\mathrm{n}$ and n'. Thus the event horizon compels not only a relationship between $\mathrm{n}$ and n', unnecessary when the system is free to irradiate energy, but also an upper limit to the value of $n$. Hence the orbiting system behaves as a whole like a black hole if its space size is smaller than that enabling the black hole condition of its total mass. In conclusion, it is possible to conceive a system that does not irradiate gravitational waves provided that its whole density fulfils that of Equation (25).

\section{THE LIGHT BEAM BENDING}

An interesting and straightforward extension of the considerations so far carried out concerns the behaviour of a quantum gravitational system at and beyond the distance characterizing the black hole condition; the Equation (7) are in particular useful for this purpose. Strictly speaking, the approach leading to the Equation (6) does not necessarily require a bound state; even under an attractive potential, the negative sign of $\varepsilon_{\mathrm{G}}$ is due indeed to the choice of quantum numbers 1 of angular momentum such that $1 \leq \mathrm{n}-1$. Yet considering for instance $1=\mathrm{n}$, means describing an open two body system. The case of interest here is that where one particle comes from minus infinity and proceeds to infinity along a curved trajectory determined by its interaction with the source of the attractive field $\varphi$. Owing to its importance, the light beam bending in a gravitational field is examined here although already introduced in a previous paper (Tosto, 2013b); it seems indeed significant to propose a further contribution to this topic exploiting purposely the results obtained in this study only, to confirm their validity and to gain further physical information on the link between quantum mechanics and relativity.

The reason why the light beam should curve its path when interacting with a gravitational source is easily explained. First of all, the photon interacts with the gravity field; otherwise its incapability of escaping from the event horizon of a black hole would be unexplained (Tosto, 2013b). Moreover a free photon moves in the vacuum with linear motion at constant speed $c$; if however it interacts with a field, then by definition its motion is perturbed. In particular is perturbed its momentum. Since $c$ is a constant, the only possible perturbation of the momentum can concern the direction of motion: The unperturbed linear path turns in the presence of the field into a perturbed bent path. Eventually if the photon interacts with the gravity field, then the propagation rate of the latter must necessarily be equal to that of the former; otherwise a photon running away faster than the field wouldn't "feel" the field and couldn't interact. This conclusion has been in effect inferred in the previous section. The black hole behaviour of the gravitational system is essential for the 
present purposes, the starting points are the quantum Equation (7) of $\Delta \mathrm{r}$ and $\varepsilon_{\mathrm{G}}$.

As the photon of energy $\varepsilon$ interacts with the gravity field, it can be regard like a particle of mass $m_{1}=\varepsilon / \mathrm{c}^{2}$; thus is now this $m_{1}$ the partner of the orbiting system of total mass $\mathrm{M}$ and reduced mass $\mu$. Rewrite identically the equation (8) as:

$$
\varepsilon_{\text {ang }}=\frac{1(1+1)}{n^{2}}\left|\varepsilon_{G}\right|=\frac{1(1+1)}{n^{2}} \frac{\Delta r_{b h}}{\Delta r}\left|\varepsilon_{b h}\right|
$$

because $\varepsilon_{G} / \varepsilon_{b h}=\Delta r_{b h} / \Delta r$ at any $\Delta \mathrm{r}$. So:

$$
\varepsilon_{\mathrm{ang}}=\xi\left|\varepsilon_{\mathrm{bh}}\right| \quad \xi=\frac{1(1+1)}{\mathrm{n}^{2}} \frac{\Delta \mathrm{r}_{\mathrm{bh}}}{\Delta \mathrm{r}}
$$

A bent path, even not necessarily closed, is consistent in principle with the existence of a local angular momentum; so the parameter $\xi$ must be purposely determined in order to describe the angular momentum of $\mathrm{m}_{1}$, identifiable in effect by the term $1(1+1)$ of the

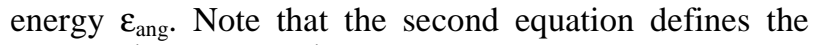
amount $\xi \Delta$ r; so let $\xi$ have consequently the physical meaning of an angle $\delta \phi$ delimiting an arc $\delta s=s_{2}-s_{1}$ $=\Delta \mathrm{r} \delta \phi$ of circumference of given radius $\Delta \mathrm{r}$, to which indeed corresponds the arc length $\delta s=1(1+1) \Delta \mathrm{r}_{\mathrm{bh}} / \mathrm{n}^{2}$. The boundary coordinates $\mathrm{s}_{1}$ and $\mathrm{s}_{2}$ define on the circumference the position of two tangents that schematize the path travelled by a photon asymptotically free at $-\infty$ and $+\infty$ : The photon coming from minus infinity approaches the circumference of radius $\Delta \mathrm{r}$ along a straight path till to the tangent point $\mathrm{s}_{1}$, then it proceeds on the circumference along the curved arc $\Delta \mathrm{r} \delta \phi$ until the point $s_{2}$, then it continues its run along a straight path from the tangent point $s_{2}$ to infinity. Of course $\delta \phi$ is also the angle between the tangents themselves, i.e., it represents the gravitational field driven deviation of the linear path of the free photon. All this requires simply assuming $\xi=\delta \phi$. Hence:

$$
\delta \phi=\frac{1(1+1)}{n^{2}} \frac{\Delta \mathrm{r}_{\mathrm{bh}}}{\Delta \mathrm{r}} \quad \delta \phi=\delta \phi(1 \geq \mathrm{n})
$$

As expected $\delta \phi$ is proportional to the local angular momentum via $1(1+1)$; the second equation emphasizes that the bending angle corresponds to an unbound quantum state of the system. Despite the large value of $n$ calculated in the Equation (9) for a bound system, it is reasonable to calculate $\delta \phi$ for the ground unbound state of the open system of interest here; to $\mathrm{n}=1$ corresponds therefore $1=1$, i.e., the ground value of perturbation driven angular momentum that governs the bending effect. One finds thus Equation (33):

$$
\delta \phi=2 \frac{\Delta \mathrm{r}_{\mathrm{bh}}}{\Delta \mathrm{r}}=\frac{4 \mathrm{GM}}{\mathrm{c}^{2} \Delta \mathrm{r}}
$$

In effect this is the well known Einstein result of light beam bending in the presence of the gravity field $\mathrm{M}$, which is here the total mass of the bodies constituting the orbital system. Of course the minimum distance $\Delta \mathrm{r}$ between photon and gravity source is replaced here by the radius of the circle that determines the size of the orbiting system and thus the arc of circumference corresponding to the angle $\delta \phi$; yet this is acceptable, because for small angles any curved trajectory can be locally approximated by an appropriate osculating circumference.

\section{DISCUSSION}

The physical nature of the gravitational waves is explained in the general relativity as the existence of ripples of the space-time: The gravity field equations admit indeed a wave-like solution. In the present quantum model, instead, the origin of the gravitational waves relies entirely on quantum considerations based on the space-time uncertainty of the Equation (1); the waves are simply the radiative decay of an orbiting quantum system formally similar to the Bohr model of atom. It is clear why circular orbits have been concerned to simplify as much as possible the mathematical approach; the essential point of the model, i.e., the quantum origin of the gravitational waves, appears conceptually clear even in this simple case without need of unnecessary complications. Actually it is easy to extend the naïve model of circular orbits to the elliptic orbits where one more parameter is necessary, i.e., the eccentricity e also introduced in a natural way in Equation (31); yet the fact that even the crudest approximation of circular orbits is enough to find a quantum result that agrees with that of relativity, ensures that the present starting point to tackle the problem is substantially correct. Although the model aims primarily to provide a reasonable quantum explanation of the gravitational waves, some crucial points also emerged during its formulation deserve being further highlighted. One of these points is to explain why the Bohr atom is stable whereas the planetary gravitational systems tend to shrink with emission of gravitational waves. The explanation does not rely on either form of the potential energy, but on the number $n$ of allowed quantum states 
compatible with the size scale of either system: Is not surprising the tendency of gravitational systems to behave similarly to the atomic systems, indeed holds anyway their propensity to the most stable configuration allowed by the fundamental laws of nature; yet the ability of the former to decay down to the ground state is more striking and evident than that of the latter just because of their different numbers $n$ of allowed quantum states. The importance of $\mathrm{n}$ appears once accepting that the physical laws of our universe are governed by the concept of space-time uncertainty, even though formulated in its most agnostic form and without need of further "ad hoc" hypotheses. Masses and uncertainty ranges of atom systems are such that $n$ is of the order of magnitude of a few units only; in fact large values of $n$ would mean abandoning the quantum world to attain the classical limit, in agreement with the correspondence principle. Instead $\mathrm{n}$ of planetary systems amounts to several powers of ten, as previously shown and in agreement with the fact that in fact the macroscopic structures fall in the realm of classical physics. In this short statement there is the double essence (i) of the different strengths of the electromagnetic and gravitational interactions that control the respective bond lengths and thus space scales and (ii) of the extraordinary success of the relativity in describing the universe dominated by the gravity force despite its classical character. For this reason the classical root of the present model has been presumed "a priori" reliable, as it seems after all confirmed.

The enhancement ingredients of the relativity are its 4-dimensional basis and covariant formulation; in the present model, instead, the enhancement ingredient is the quantum contribution provided by the Equation (1) only. As these equations have inherently 4-D character, the comparison with the relativistic formalism reveals that somehow the space-time uncertainty surrogates the concept of covariancy, although initially conceived aiming to quantum problems only; this conclusion appears validated also by the further relativistic results concerned in (Tosto, 2013b). In effect the idea of covariancy has been introduced also here, to guess a similar form of the Equation (27) and (28).

To emphasize a significant implication of the model, the sum $\mathrm{J}^{2}+2\left(\alpha_{2}-\mathrm{b}\right) \mu$ appearing in the Equation (5) deserves a short digression. Since is self-evident that the addend $\left(\alpha_{2}-b\right) \mu$ must be a square angular momentum itself, let us require that $\left(\alpha_{2}-\mathrm{b}\right) \mu=0$ for $1=0$ likewise $\mathrm{J}^{2}$ $=1(1+1) \hbar^{2}$ : It is not admissible that the angular momentum of any system can be zero or different from zero depending on the number of terms only of the series development of the interaction potential introduced in the energy equation. Therefore it must be true that $\left(\alpha_{2}-b\right) \mu= \pm 1 s \hbar^{2}$ depending on the sign of $\alpha_{2}$-b. To understand what $s$ might be, note in this result the product $1 \hbar$ times sh; i.e., also sh, likewise $1 \hbar$, should be the component of an angular momentum vector $\mathrm{S}$ along an arbitrary direction defined by the unit vector w. In effect the numerator of the last Equation (5), rewritten identically but more expressively as $\mathbf{J}^{2} \pm 21 s \hbar^{2}$, reads also as follows Equation (34):

$$
\begin{array}{ll}
1(1+1) \hbar^{2} \pm 21 s \hbar^{2}=(\mathrm{L} \pm \mathrm{S})^{2}-\mathrm{S}^{2} & \mathrm{~L}^{2}=1(1+1) \hbar^{2} \\
\mathrm{~S}^{2}=\mathrm{s}(\mathrm{s}+1) \hbar^{2} & \mathrm{~L} \cdot \mathrm{S}= \pm \mathrm{ls} \hbar^{2}
\end{array}
$$

Here we have reasonably inferred $S^{2}$ from $s$ in the same way as does $\mathrm{L}^{2}$ from 1 . On the one hand the first equation agrees with the previous statement that $\mathbf{J}$ must be included within a quantum range $\Delta \mathrm{J}$ as a consequence of the positions (2), likewise as $r$ and $\mathrm{p}_{\mathrm{r}}$ in the classical equation (3) turn into the respective uncertainty ranges $\Delta \mathrm{r}$ and $\Delta \mathrm{p}_{\mathrm{r}}$ of the quantum Equation (4). On the other hand we have made explicit what the range $\Delta \mathrm{J}$ actually is, i.e., $\Delta \mathrm{J}=\mathrm{J}_{\mathrm{L}+\mathrm{S}}-\mathrm{J}_{\mathrm{S}}=(\mathrm{L} \pm \mathrm{S})-\mathrm{S}$ : The vector $\mathrm{S}$ defines together with $\mathrm{L} \pm \mathrm{S}$ the boundaries of the angular momentum uncertainty range $\Delta \mathrm{J}$. While the first Equation (34) evidences that $(\mathrm{L} \pm \mathrm{S})^{2}-\mathrm{S}^{2}$ including both coefficients of series development anyway vanishes for $\mathrm{L}=0$, as required by the motivation of the present reasoning at the second order approximation of the potential $\varphi$, the interesting fact is that $L=0$ does not necessarily exclude $S \neq 0$; it simply entails $S^{2}-S^{2}=0$. Eventually if $1(1+1)$ is an integer, then also 21 s must be an integer itself, once more because of the same aforesaid motivation; yet this condition is fulfilled by $\mathrm{s}$ integer or even half integer, which shows that 1 and $s$ have different physical meaning. Since the former represents the angular space motion of a particle, as shown in the section 2 and in the previous examples, the latter can be nothing else but an intrinsic angular momentum of the particle itself.

For brevity the digression stops here because the spin of quantum particles is not relevant for the purposes of the present paper mostly devoted to planetary systems, in effect it has been skipped everywhere; yet this short note, although introduced here as it would be a mere marginal remark only, shows once more that the Equation (1) yield indifferently and contextually quantum and relativistic results without hypotheses additional to the concept of uncertainty only.

It is also worth noting the Einstein abandoned the concept of gravity force for that of space-time 
deformation in the presence of mass. Here instead the force appears explicitly, see for instance the first Equation (15); yet also here this force is nothing else but space-time deformation. What else otherwise could represent from a physical point of view the changes of the uncertainty ranges $\Delta \delta \mathrm{p}_{\mathrm{r}}$ due to $\Delta \delta \mathrm{r}$ and $\Delta \delta \mathrm{t}$ ? The only difference with respect to the relativity is that here the deformation of space-time does not involve the dynamical variables, but ranges of conjugate dynamical variables subjected to the requirement of uncertainty. This makes the concept of space-time deformation even more abstract than in relativity; moreover the positions (2) make useless the tensor formalism that necessarily implements assigned values of local dynamical variables, here instead disregarded since the beginning conceptually and not as a sort of approximation to simplify some calculation. The results are nevertheless consistent with that of the relativity.

A significant outcome of the model is the evidence that actually the planetary systems have a feature in common with the atomic systems, i.e., the ability of regarding emission and absorption of energy in a reversible and symmetric way. Atoms absorb or release electromagnetic waves via excitation or decay of electrons in their allowed energy levels, whereas the effective chance of energy exchange between them is subjected to the resonance condition. Also in this respect, the quantum approach prospects an analogous mechanism of emission and absorption of gravitational waves; by definition both signs are admissible for the change $\delta n$ of the number of states, which in turn determine the wavelengths and controls the signs of $\delta \Delta \varepsilon / \delta \Delta \mathrm{t}$ and $\delta \Delta \mathrm{r} / \delta \Delta \mathrm{t}$ that discriminate either chance of releasing or absorbing energy. This conclusion holds because the results of section 3 show that in fact $n$ and 1 play exactly the role of the respective quantum numbers characterizing the solution of the Schrodinger equation. Note however that while is very easy to infer the quantum angular momentum and the electron energy levels of hydrogenlike atoms via the Equation (1), it would have been really problematic to infer the gravitational waves via the wave formalism solving the appropriate Schrodinger equation; the broad spectrum of results attainable via the Equation (1) is the best proof of their wider generality. In this respect, it is interesting the fact that the quantum number 1 of non-relativistic angular momentum appearing in the Equation (34), controls the relativistic expression (33) of the light beam bending; this is the quantum signature underlying also a crucial result of the general relativity, the space-time curvature, to explain the gravity force.

Deserves attention also the fact of having inferred as a corollary of the Equation (1) even the Newton law (Tosto, 2000; 2013b), which is itself a further crucial point of the present paper; it shows that the Equation (1) are in fact a fundamental principle of nature, able to include both quantum and relativistic results. Indeed the same conceptual basis exploited in the present paper allowed obtaining as a corollary even the Hawking entropy and temperature together with the Friedmann equation, which is the fundamental theoretical basis to describe the evolution of the universe.

In this respect it is worth emphasizing a side outcome of the present model, i.e., the anti-gravity. This idea, already introduced in a previous paper (Tosto, 2013b), appears more clearly while discussing the quantum formulation of the eigenvalues of a gravitational system. When considering the signs allowed for $\alpha_{1}$ and $\alpha_{2}-b$ in the Equation (4) and (5), the attractive gravitational potential allowing the bond energy between two isolated masses results contextually compatible also with the existence of a repulsive gravitational potential between these masses. This chance opens a new scenario about the forces with which interacts the matter of our universe: The sensible results obtained via one particular choice of signs, of course that leading to the "conventional" gravity force, compels accepting also the idea of alternative unusual anti-force. The so called "fifth force", has been in effect evocated by some authors (Hayasaka and Takeuchi, 1989) to support exotic hypotheses, like the negative energy and the worm-holes (Morris et al., 1988; Hathaway et al., 2003). Moreover strange phenomena, like the so called Hutchison effect, have been also debated although in lack of firm experimental evidence. Besides the fact that no experimental test is today considered conclusive to validate these ideas, the major issue of this topic is that the theoretical frame is still unclear itself. Despite all theoretical efforts are at present carried out exclusively within a relativistic frame, further quantum outcomes in progress could hopefully contribute to clarify this challenging topic. The previous considerations on the signs of the terms of series development of the interaction potential $\varphi$, whose particular choice allowed inferring the Newton potential as first order approximation, are under investigation. Indeed two significant hints appear in this quantum model. One concerns once again the successful chance of defining the first coefficient $\alpha_{1}$ both as $\pm \mu \mathrm{GM}$ and $\pm \mathrm{Ze}^{2}$, the former leading to the gravity force the latter to the Coulomb force, that describe likewise a planetary system 
and the Bohr atom: So in a sense it is not surprising that the familiar attractive/repulsive character of the latter corresponds to an analogous attractive/repulsive ability of the former. The formal symmetry between the electromagnetic and gravitational interaction has been already remarked, see for instance the Equation (13) where still appears $\hbar$; despite the enormous difference of strength and physical meaning, the former obeys the Maxwell equations the latter does not, the quantum mechanism of emission of electromagnetic radiation or gravitational waves reveals an amazing similarity. Something else must be hidden in this indication, which cannot be merely accidental. The second hint concerns the non-trivial fact that even the Coulomb law is a first order approximation only of a more complex interaction, as in effect it has been found in (Tosto, 2004). Also note in this respect that the Equation (33) has been inferred considering the light beam deflection under the usual attractive potential, in which case the light beam tends to wrap around the gravity source; however the hypothesized antigravity potential, if really still existing and active somewhere in the universe, stimulates in principle the question about how it could deflect a light beam: May be in an opposite way, i.e. similarly to the behaviour of two charges of the same sign.

In the previous papers and in the present one, the quantum approach goes deep into the general relativity realm without necessity of requiring extra-dimensions to introduce the quantization condition into the model of gravitational system. Of course the present model does not exclude their physical reality, waiving the extradimensions is simply consequence of the basic idea followed here: Rather than attempting to modify the relativity in order to make it compliant with the quantum theory, the model has shown that in fact the former is a branch of the latter once accepting the idea of space-time uncertainty as a common root. Indeed the present model does not propose an alternative path to the gravitational waves still in the frame of some relativistic theory born after the early Einstein formulation; the paper aims instead to propose a new "ab initio" approach based on the Equation (1) only and to check the agreement of the quantum results with the relativistic ones. It must be noted in this respect that the relativity alone is incomplete if not merged with the quantum theory; for instance it cannot account for the important effects like the Hawking entropy or the vacuum polarization, as instead the present model does.

The quantum instability of the orbiting systems has at least three further implications, whose discussion needs however an introductory remark of general character. In
(Tosto, 2013b), for instance, the link between local momentum $\mathrm{p}$ and energy $\varepsilon$ of a free particle moving at rate $\mathrm{v}$ has been found in the form $\Delta p=\mathrm{v} \Delta \varepsilon / c^{2}$; the quantum uncertainty ranges show therefore that the functional relationship between $\mathrm{p}$ and $\varepsilon$ is $p=\mathrm{v} \varepsilon / c^{2}$. This result, obtained exploiting the Equation (1) only, agrees with but it is not identical replica of the special relativity; here $\mathrm{p}$ and $\varepsilon$ are random local variables, in principle unknown and unknowable, included in the respective uncertainty ranges whose sizes are unknown and unknowable as well. Yet, despite this agnostic starting point, previous papers show how to obtain both relativistic and quantum results; in this paper, significant examples are the Equation (23) and (24) on the one side and the Equation (34) on the other side. So the statement that the present model is not mere replica of the relativity has a well defined physical meaning: The equations have the same form of the relativistic ones, of course, but fully quantum character because they inherently contain the essential concept of uncertainty. These considerations suggest new hints to the cosmology and to the relativity itself.

The first implication concerns the chance of energy transfer within the universe via gravitational waves. A possible mechanism envisages an orbiting system emitting waves that once intercepted by an analogous system far apart excite this latter to a higher energy quantum level; the idea is well known, any system absorbs the waves it is able to emit. The similarity with the analogous mechanism involving photons and electron energy levels agrees with and supports the idea of "graviton", i.e. quantum of wave energy introduced through $\delta$ at the end of the section 4 . Very high values of $n$ mean small spacing between gravitational quantum levels, so that $n \gg>1$ and $\delta n \gg>1$ can be approximately regarded as continuous variables; then appropriate $\delta n$ and $\mathrm{n}$ can realistically fulfil the resonance condition between two orbiting quantum systems far apart. Despite the tiny interaction effect measurable according to the results of the section 5 , the huger space scale of the cosmic systems involved and the large number of bodies orbiting around or accelerated by the field of other masses in the whole universe suggest the actual chance of relevant amounts of energy exchanged with this coupling mechanism.

Recall now the reasoning introduced when describing the light beam bending: The motion of a test mass perturbed by a field implies a local angular momentum and thus a local angular velocity $\omega$ and curvature radius $\Delta \mathrm{r}$. The second implication of the model concerns just 
the light beam bending, which from a gravitational point of view has been described by photons of energy $\varepsilon$ and equivalent mass $\mu=\varepsilon / \mathrm{c}^{2}$. Thus: Are bent light beams able to irradiate themselves gravitational waves? If so, then their wavelengths should increase in the presence of a perturbing gravity field; this effect is similar to the redshift of a photon climbing radially outwards of the field. In this specific case the relativity describes a symmetric process, i.e., blueshift is expected when a photon falls towards the gravity source; it is not surprising therefore that both redshift and blueshift could also occur even when photons emit or absorb gravitational waves via the coupling mechanism previously introduced. In fact have been experimentally observed bursts of $\gamma$-rays of the order of $10^{47} \mathrm{~J}$, which thus correspond to a total equivalent mass of the order of $10^{30} \mathrm{Kg}$ similar to that of the sun; as these figures are comparable to that of orbiting solid bodies, it follows that at least in principle even the light beam bending could significantly contribute to the total energy balance of the gravitational waves spreading throughout the universe.

It follows by consequence the third implication of the quantum model, i.e. the ability of photons to interact indirectly with matter via gravitational waves: Once regarding orbiting/accelerated matter and bent light beams as potential emitters/absorbers, holds for both the possibility of positive or negative $\delta$, they in fact exchange energy. Also this kind of long distance interaction could be significant because of the huge number of systems prospectively involved within the whole universe.

This picture of interactions, although qualitative, reveals a universe more interconnected and complex than that expected considering the direct effects of the gravity only. One aspect of the research in progress about these long-range interactions aims to verify these ideas and quantify the aforesaid effects, for instance calculating the probability of gravitational wave induced transitions between orbiting quantum levels. All these effects involve well known topics typical of the quantum mechanics; so no conceptual restriction hinders in principle extending further the results hitherto exposed in order to better understand the physics of the gravitational waves, in particular their interaction with light and matter. Crucial appear instead the conceptual limits posed by the quantum theory itself. In other words further information on the physics of the gravitational wave is achievable, but within the limits of the quantum knowledge we can afford. This compelling condition, consequential in a quantum approach only, deserves more attention.
The previous results strongly suggest that both relativity and quantum theory have a common fundamental root, the uncertainty i.e., our hopeless inability to know everything with arbitrary accuracy: The Equation (1) represent a sort of insurmountable barrier that defines the essential information we can afford about the events allowed in nature. It is really difficult to think that such a limit of the quantum world should not hold also in the relativistic realm. Just to this aspect of the model is devoted a closing remark.

The quantum mechanical approach hitherto carried out via the Equation (1) could seem less complete than that carried out via relativistic approach; for instance we haven't concerned the polarizations " $x$ " and " + " of the gravitational waves, rather a simple energy balance on the gravitational system only was enough to explain its instability. Of course further investigation on these points, in this paper waived uniquely for brevity, is presently in progress. This omission is however mostly due to the priority attention that must be paid to the limits posed by the quantum theory on the physical observables: Any classical approach allows in principle calculating everything, the commutation rules of quantum operators discriminate instead which amounts really correspond to physical eigenvalues.

\section{CONCLUSION}

The quantum approach introduced by the present model poses new problems about the science of the gravitational waves. The quantum theory was refused by Einstein because considered imperfect ("God does not play dice"), incomplete (e.g., $\mathrm{J}^{2}$ and $\mathrm{J}_{\mathrm{z}}$ only have physical meaning), non-local (EPR paradox and "hidden variables") and non-real ("The moon exists even though nobody observes it").

The relativity defines in detail several properties of the gravitational wave, e.g., its " $\mathrm{x}$ " and "+" polarizations resulting from the quadrupole approximation to solve the field equations as a perturbation of the flat Minkowski space-time. In effect the classical origin of the relativity does not put in principle limits to the amount and kind of quantities calculable; the quantum theory instead does, e.g., nobody could dare measuring $J_{z}$ and $J_{x}$. So at the end of this study is legitimate the question about how, in general, the quantum standpoint could modify also the commonly acknowledged experimental frame to validate the theoretical outcomes of the relativity. A possible task is to examine carefully the true physical motivation of the unsuccessful attempts so far carried out to detect directly the waves, despite an ample amount of 
observations provides indirect proofs of their existence (Weisberg et al., 1981). Is this experimental difficulty really due to the tiny contraction/dilation effects inherent the weak intensity of the waves only? or perhaps are we attempting to measure some unphysical quantum effect?

\section{REFERENCES}

Blandford, R.D. and R. Narayan, 1992. Cosmological applications of gravitational lensing. Annual Rev. Astronomy Astrophys., 30: 311-358. DOI: 10.1146/annurev.aa.30.090192.001523

Clayton, D., 2007. Hoyle's equation. Science, 318: 18761877. DOI: 10.1126/science. 1151167

Cohen, N., 1988. Gravity's Lens: Views of the New Cosmology. 1st Edn., Wiley and Sons, New York, ISBN-10: 0471632821, pp: 237.

Dinculescu, A., 2013. The cosmic microwave background: A strange characteristic. Am. J. Space Sci., 1: 61-62. DOI: 10.3844/ajssp.2013.61.62

Duff, M.J., 1996. M-Theory (the theory formerly known as strings). Int. J. Modern Phys. A, 11: 5623-5642. DOI: $10.1142 / \mathrm{S} 0217751 \mathrm{X} 96002583$

Hartle, J.B. and S.W. Hawking, 1976. Path-integral derivation of black hole radiance. Phys. Rev., D, 13: 2188-2203. DOI: 10.1103/PhysRevD.13.191

Hathaway, G., B. Cleveland and Y. Bao, 2003. Gravity modification experiment using a rotating superconducting disc and radio frequency fields. Phys. C, 385, 4: 488-500. DOI: 10.1016/S09214534(02)02284-0

Hayasaka, H. and S. Takeuchi, 1989. Anomalous weight reduction on a Gyroscope's right rotations around the vertical axis on the Earth. Phys. Rev. Lett., 63: 2701-2704. DOI: 10.1103/PhysRevLett.63.2701

Jungmann, G., M. Kamionkowski and K. Griest, 1996. Supersymmetric dark matter. Phys. Reports, 267: 195-195. DOI: 10.1016/0370-1573(95)00058-5

Landau, L.D. and E.M. Lifshitz, 1987. The Classical Theory of Fields. 1st Edn., Pergamon Press.

Leonhardt, U. and H. Paul, 1995. Measuring the quantum state of light. Progr. Quant. Electr., 19: 89130. DOI: 10.1016/0079-6727(94)00007-L

Levanda, M. and V. Fleurov, 2001. A. Wigner quasidistribution function for charged particles in classical electromagnetic fields. Annals Phys., 292: 199-231. DOI: 10.1006/aphy.2001.6170

Lidsey, J.E., D. Wands and E.J. Copeland, 2000. Superstring cosmology. Phys. Reports, 337: 343492.
Marck, J.A. and R. Lasota, 1997. Relativistic Gravitation and Gravitational Radiation Inclusive CD-ROM. 1st Edn., Cambridge University Press, Cambridge, ISBN-10: 0521590655, pp: 475.

Milonni, P.W., 1994. The Quantum Vacuum: An Introduction to Quantum Electrodynamics. 1st Edn., Academic Press, Boston, ISBN-10: 0124980805, pp: 522.

Morris, M., K. Thorne and U. Yurtsever, 1988. Wormholes, time machines and the weak energy condition. Phys. Rev. Lett., 61: 1446-1449. DOI: 10.1103/PhysRevLett.61.1446

Peskin, M.E. and D.V. Schroeder, 1995. An Introduction to Quantum Field Theory. 1st Edn., Addison-Wesley Publishing Company, Reading, ISBN-10: 0201503972, pp: 842.

Polchinski, J., 1998. String Theory. 1st Edn., Cambridge University Press, New York, ISBN-10: 0521633125.

Polchinski, J., 2001. String Theory Vol. II: Superstring Theory and Beyond. 1st Edn., Cambridge University Press.

Rothman, T. and S. Boughn, 2006. Can gravitons be detected? Foundat. Phys., 36: 1801-1825. DOI: 10.1007/s10701-006-9081-9

Seeger, P.A., W.A. Fowler and D.D. Clayton, 1965. Nucleosynthesis of heavy elements by neutron capture. Astrophys. J. Suppl, 11: 121-66. DOI: 10.1086/190111

Thorne, K.S., 1973. Relativistic shocks: The taub adiabat. Astrophys. J., 179: 897-907. DOI: $10.1086 / 151927$

Tosto, S., 2000. An analysis of states in the phase space: From quantum mechanics to general relativity.

Tosto, S., 2004. An analysis of states in the phase space: The relativistic many electron atom. Recent Res. Dev. Phys., 5: 1363-1422.

Tosto, S., 2012. Quantum uncertainty and relativity. Progress Phys., 2: 58-81.

Tosto, S., 2013a. Space-time uncertainty and cosmology: A proposed quantum model of the universe. Progress Phys., 4: 3-24.

Tosto, S., 2013b. Quantum standpoint for a more understandable universe. Am. J. Space Sci., 1: 2232. DOI: $10.3844 /$ ajssp.2013.22.32

Weisberg, J.M., J.H. Taylor and L.A. Fowler, 1981. Gravitational waves from an orbiting pulsar. Scient. Am., 245: $\quad 74-82$. 10.1038/scientificamerican1081-74

Will, C.M., 1998. Bounding the mass of the graviton using gravitational-wave observations of inspiralling compact binaries, Phys. Rev. D, 57: 2061-2068. DOI: 10.1103/PhysRevD.57.2061 